\title{
Mathematical modeling of monoclonal conversion in the colonic crypt
}

\author{
Alexander G. Fletcher ${ }^{\mathrm{a}, \mathrm{b}, *}$, Christopher J.W. Breward ${ }^{\mathrm{c}}$, S. Jonathan Chapman ${ }^{\mathrm{d}}$ \\ a Centre for Mathematical Biology, Mathematical Institute, University of Oxford, 24-29 St Giles', Oxford OX1 3LB, UK \\ ' ${ }^{\mathrm{b}}$ Oxford Centre for Integrative Systems Biology, Department of Biochemistry, University of Oxford, South Parks Road, Oxford OX1 3QU, UK \\ ' Oxford Centre for Collaborative Applied Mathematics, Mathematical Institute, University of Oxford, 24-29 St Giles', Oxford OX1 3LB, UK \\ ' Oxford Centre for Industrial and Applied Mathematics, Mathematical Institute, University of Oxford, 24-29 St Giles', Oxford OX1 3LB, UK
}

\section{A R T I C L E I N F O}

\section{Article history:}

Received 5 March 2011

Received in revised form

17 November 2011

Accepted 13 January 2012

Available online 23 January 2012

\section{Keywords:}

Crypt

Stem cells

Monoclonal conversion

Cancer

\begin{abstract}
A B S T R A C T
A novel spatial multiscale model of a colonic crypt is described, which couples the cell cycle (including cell division) with the mechanics of cell movement. The model is used to investigate the process of monoclonal conversion under two hypotheses concerning stem cell behavior. Under the first hypothesis, 'stem-ness' is an intrinsic cell property, and the stem cell population is maintained through asymmetric division. Under the second hypothesis, the proliferative behavior of each cell is governed by its microenvironment through a biochemical signalling cue, and all cell division is symmetric. Under each hypothesis, the model is used to run virtual experiments, in which a harmless labeling mutation is bestowed upon a single cell in the crypt and the mutant clonal population is tracked over time to check if and when the crypt becomes monoclonal. It is shown that under the first hypothesis, a stable structured cell population is not possible without some form of population-dependent feedback; in contrast, under the second hypothesis, a stable crypt architecture arises naturally. Through comparison with an existing spatial crypt model and a non-spatial stochastic population model, it is shown that the spatial structure of the crypt has a significant effect on the time scale over which a crypt becomes monoclonal.
\end{abstract}

(c) 2012 Elsevier Ltd. All rights reserved.

\section{Introduction}

Large-bowel or colorectal cancer accounts for $13 \%$ of all cancers in the United Kingdom, with around 35,300 new diagnoses and 16,000 deaths occurring each year (Cancer Research UK, 2010). Colorectal cancer is predominantly a disease associated with old age, with $80 \%$ of diagnoses being made in patients over the age of 60 . As a result of both longer life expectancy and declining fertility rates, the proportion of people in this age group is growing faster than any other. Colorectal cancer is therefore likely to rise in prevalence (World Health Organization, 2008).

Colorectal cancers originate from the epithelium that covers the luminal surface of the intestinal tract. This epithelium renews itself more rapidly than any other tissue (Ross et al., 2003). This process of renewal requires a coordinated programme of cell proliferation, migration and differentiation, which begins in the tiny crypts of Lieberkühn that descend from the epithelium into the underlying connective tissue. It is generally believed that tumourigenesis occurs as a consequence of changes that disrupt

\footnotetext{
* Corresponding author at: Centre for Mathematical Biology, Mathematical Institute, University of Oxford, 24-29 St Giles', Oxford OX1 3LB, UK.

Tel.: +441865 273525; fax: +441865273583.

E-mail address: alexander.fletcher@maths.ox.ac.uk (A.G. Fletcher).
}

normal crypt dynamics. Identifying the mechanisms that govern crypt dynamics is therefore essential to understanding the origins of colorectal cancer.

In this paper we describe a novel spatial multiscale model of a colonic crypt, which couples the cell cycle (including cell division) with the mechanics of cell movement. We will use our model to evaluate the immortal stem cell hypothesis and the stem cell niche hypothesis by studying the process of monoclonal conversion under each hypothesis. The modular nature of this model allows us to easily investigate different assumptions concerning stem cell behavior. In each case, we use the model to run virtual experiments in which a harmless labeling mutation is bestowed upon a single stem cell in the crypt, and the mutant clonal population is tracked over time to check if and when the crypt becomes monoclonal. This is impossible to perform in vivo with current experimental techniques.

\subsection{Crypt architecture}

A schematic diagram of a normal healthy colonic crypt is shown in Fig. 1. Stem cells, located at the bottom of the colorectal crypt, divide to produce transit-amplifying cells that migrate up the crypt wall (Barker et al., 2007). As the cells migrate up the crypt they undergo four to six rapid divisions (Potten et al., 2003) before they stop dividing and differentiate terminally into colonocytes, 


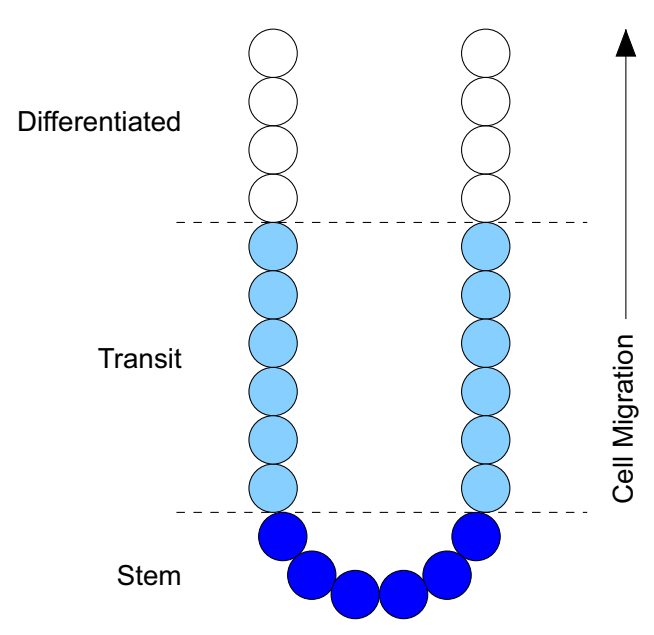

Fig. 1. Diagram indicating the spatial location of the stem, transit amplifying and differentiated cell compartments within the crypt.

enteroendocrine cells or Goblet cells (Brittan and Wright, 2004). Once at the top of the crypt, cells either undergo apoptosis or are shed into the lumen and transported away (Giles et al., 2003; Nowak et al., 2002).

Crypts are generated in adult mice by longitudinal crypt fission, which is likely to start at the bottom of the crypt, where the stem cells reside. This spatial coincidence has led to the proposal that crypt generation is linked to stem cell behavior. Crypt extinction is also possible and occurs if all actual and potential stem cells are eliminated, for example after exposure to radiation or cytotoxic drugs (Loeffler et al., 1997).

Since most data on the tissue renewal cycle come from experimental studies of the murine small intestine, we will concentrate on this type of crypt. Adult murine crypts contain about 250 cells, of which 150 are proliferating, producing about 200-300 cells every day (Potten and Loeffler, 1990). Epithelial cell turnover is rapid and essentially all cells except stem cells are replaced within a week. In the murine small intestine, it takes 2-3 days for a cell to migrate up from the base of the crypt to its apex (Okamoto and Watanabe, 2004). All crypt cells, excluding stem cells, will be renewed over this period. The stem cells divide every 12-32 h with a mean $24 \mathrm{~h}$ (Potten et al., 2003; Li et al., 1994). The transit cell population has an estimated cell-cycle time of 11-12 h (Giles et al., 2003; Loeffler et al., 1986; Meineke et al., 2001).

Based on indirect evidence, there have been various estimates for the number of stem cells in a murine small intestinal crypt, ranging from 1 to about 16 (Boman et al., 2001; Marshman et al., 2002; Potten and Loeffler, 1990), with a mean of around 6-8. It has been proposed that this number varies slowly with time (Loeffler and Grossmann, 1991) and that observed crypt size distributions are proportional to the distribution of stem cells per crypt (Loeffler et al., 1997). Despite the central role of the stem cells in maintaining the integrity of the intestinal epithelium, for many years their precise number and location within the crypt remained unclear, mainly due to a lack of unique marker genes and the absence of stem-cell assays. Recently, however, Barker et al. (2007) were able to show that the Wnt target gene Lgr5 is a marker for stem cells in the small intestine and colon.

\subsection{Monoclonal conversion}

It has been shown experimentally that, over time, the progeny of a single stem cell within a crypt can take over the entire crypt. This process has been termed monoclonal conversion (McDonald et al., 2006), since the resulting crypt consists of a single clonal population. Monoclonal conversion is important in the context of colorectal carcinogenesis as clonal crypts seem able to expand into patches through a process called crypt fission. The time scale over which a crypt becomes dysplastic is related to the time scale over which monoclonal conversion occurs and the rate at which somatic mutations occur within the crypt. It has been proposed that monoclonal, mutant crypts constitute the earliest stage of colorectal adenomas (Preston et al., 2003). These monocryptal lesions can expand further by crypt fission (Greaves et al., 2006).

The spread of cellular clones induced by somatic mutation has been studied in murine intestinal crypts using the effect of mutation of the D1b-1b locus in stem cells induced by the mutagen ethylnitrosurea (ENU), which can cause mutations to occur in the glucose-6-phosphate dehydrogenase (G6PD) gene (Winton et al., 1988; Winton and Ponder, 1990; Loeffler et al., 1993). Studies in mice that have received an injection of ENU initially show a G6PD deficiency in only some crypt cells, but over time the entire crypt can convert to being wholly deficient (Park et al., 1995). More recent studies have shown similar results with the induction of crypt-restricted metallothionein by ENU (Cook et al., 2000), and with the induction of mitochondrial DNA (mtDNA) mutations resulting in a cytochrome $c$ oxidase deficiency (McDonald et al., 2006).

Monoclonal conversion was demonstrated recently by Barker et al. (2007) as part of their paper in which the Wnt target gene Lgr5 was found to be a marker for stem cells in the small intestine and colon. Using an activated reporter (LacZ) as a genetic marker for Lgr5 ${ }^{+}$cells and their progeny, which enabled lineage tracing, the authors found that Lgr5 is expressed only in a few cells located at the base of each crypt. Five days after induction of LacZ activity, the authors observed that $\mathrm{Lgr}^{+}$cells were still restricted to the bottom of most crypts, with only a few entirely stained crypts. Thereafter, the relative number of entirely stained crypts increased. From these observations we may infer that the progeny of a small number of stem cells (in some observations by Barker et al., 2007, a single cell), located at the base of the crypt, eventually populate the entire crypt.

\subsection{The stem cell niche hypothesis}

Stem cells are characterized by their ability to generate on average one replacement stem cell and one differentiated cell at each division with no apparent limit, thus simultaneously produce a continuous output of differentiated cells while maintaining their own population. Two complementary hypotheses have been proposed to account for this ability: the 'immortal stem cell' hypothesis and the 'stem cell niche' hypothesis.

Under the 'immortal' stem cell hypothesis, the property of being a stem cell is intrinsic to the cell. Stem cells divide asymmetrically to produce one stem cell and one non-stem cell on each division. Asymmetric cell division has been demonstrated for a number of specific cell types, for example, epidermal stem cells (Lechler and Fuchs, 2005). It has also been suggested that the immortal stem cell hypothesis could be realised by stem cells dividing symmetrically, either to produce two identical stem cells or two identical differentiated daughter cells, with equal probabilities of each situation occurring to ensure the correct average behavior. This generalization overcomes the inflexibility of constant self-renewing and differentiation rates and allows for true self-renewal of the stem cell pool with respect to cell numbers. A problem with this first hypothesis is that it completely discounts a cell's microenvironment, which is known to play an important role in the determination of cellular fate.

The second proposed hypothesis is that of a 'stem cell niche'. Here, a particular localized region or microenvironment instructs cells to function as stem cells in vivo through mechanical and biochemical signalling cues. The concept of a stem cell niche was 
first proposed in the context of haematopoietic stem cells in bone marrow (Schofield, 1978). The evidence for stem cell niches in various tissues has been reviewed by Spradling et al. (2001). In the case of the colon, we use the term microenvironment to denote a single crypt.

Two distinct mechanisms have been suggested for niche operation: the 'lineage' mechanism and the 'population' mechanism (Roeder and Lorenz, 2006). In a lineage mechanism, the division of individual stem cells and the fate of individual daughter cells are controlled by their microenvironment. For example, a stem cell in such a niche might contact the stroma or underlying tissue asymmetrically and orientate its division plane to ensure that only one daughter inherits adhesive contacts with the basement membrane. The daughter inheriting these contacts is held in the niche, where it will continue to be maintained as a stem cell, whereas the other daughter becomes untethered and relocates away from the stromal cells and their signals. Thus, a normal lineage niche operates in accordance with the 'immortal' stem cell hypothesis.

In a population mechanism, both daughter cells may remain as stem cells or both may differentiate. A stem cell does not have to 'know' its developmental direction: it acts as a stem cell, taking one of the two alternative developmental fates as dictated by its microenvironment. These two mechanisms can be distinguished by lineage studies in which single labeled stem cells and their progeny are followed: the number of labeled stem cells will remain unchanged by a lineage mechanism, but a population mechanism will eventually homogenize the labeling of all the niche's stem cells. It has also been proposed that in addition to 'actual' stem cells, differentiated cells may be 'potential' stem cells in the sense that, under appropriate local chemical cues, they may de-differentiate. This concept, explicitly assuming a potential reversibility of stem cell development, was originally proposed for the intestinal crypt by Potten and Loeffler (1990).

If a stem cell niche does indeed exist in the colonic crypt, then the Wnt signalling pathway is a prime candidate for being the niche 'controller'. Wnt signalling has been shown to play an important role in regulating normal crypt dynamics (Sansom et al., 2004; Korinek et al., 1998). This pathway is initiated when extracellular Wnt factors bind to specific receptors on the cell surface. This triggers a cascade of intracellular reactions, leading eventually to the transcription of Wnt target genes coding for proteins involved in cell-cycle control, migration and apoptosis. Mutations in key components of the Wnt pathway, such as APC and $\beta$-catenin, have been shown to be the first step in colorectal carcinogenesis in the majority of colorectal cancers, including most hereditary cases (Ilyas, 2005). It has been proposed that the stem cell niche in the colonic crypt is regulated by a spatial gradient of source of extracellular Wnt factors along the crypt axis (van de Wetering et al., 2002).

In order to detect a stem cell niche, we must be able to distinguish between cells. Until recently, the lack of a colonic stem cell marker meant that mutagenesis studies were required to detect stem cell expansion or extinction. Williams et al. (1992) follow the time course of mutation-induced phenotypic changes after a single dose of mutagen, and finds an increase in frequency of completely mutated crypts. This result was used as evidence for the existence of a stem cell niche in the crypt, with random cell loss after stem cell division. A small difference in the number of crypt stem cells that occupy the niche explained the large difference in the time taken for phenotypic changes in the murine large and small intestines after administration of a single dose of mutagen.

Stem cells can also be distinguished from each other by looking at methylation patterns. Under the immortal stem cell hypothesis, we would expect to see a wide variety of methylation patterns persisting with time. In contrast, under the stem cell niche hypothesis, crypts should eventually become homogeneous (for one or other marker state), because of random stem cell extinction and expansion. Shibata and co-workers have used nonspatial stochastic modeling (Kim and Shibata, 2002; Ro and Rannala, 2001; Yatabe et al., 2001) to demonstrate that the latter hypothesis is consistent with experimental data from stochastic methylation studies, in which the progeny from a single stem cell periodically takes over the whole crypt. This phenomenon is possible if there is more than one stem cell per crypt and stem cells can divide symmetrically. Mosaic crypts generated in such experiments, or by somatic mutation in vivo, progress with time to become either all labeled or all unlabeled. Such rapid equilibration is the expected result when a niche is maintained by a population mechanism rather than by asymmetric stem cell divisions (Spradling et al., 2001). The recent identification of the colonic stem cell marker Lgr5, and experimental findings on the timecourse of Lgr5 $^{+}$cell populations in crypts, by Barker et al. (2007) have confirmed these observations. In conclusion, the available evidence is compatible with the assumption of a stem cell niche employing some form of population mechanism, although the precise nature of how this niche operates remain to be elucidated.

A problem with the studies described above is that the mutagenic manipulations required to observe crypt heterogeneity may alter the normal behavior of stem cells. For example, stem cells that are normally immortal may alter their behavior to repair the damage induced by mutagenesis. In this case, the observed niche behavior reflects abnormal tissue damage rather than normal crypt maintenance. Moreover, the experimental manipulations used in murine stem cell studies are impractical for human studies. Mathematical and computational modeling provides a tool for testing various hypotheses about stem cell behavior in the crypt while avoiding these types of problems.

\section{Model development}

In this section we describe a spatial model of a colonic crypt which couples the cell cycle and cell division with the mechanics of cell movement. A diagram illustrating the coupling is presented in Fig. 2.

\subsection{Mechanical model}

We follow broadly the mechanical model of Paulus et al. (1992), Meineke et al. (2001) and van Leeuwen et al. (2009),

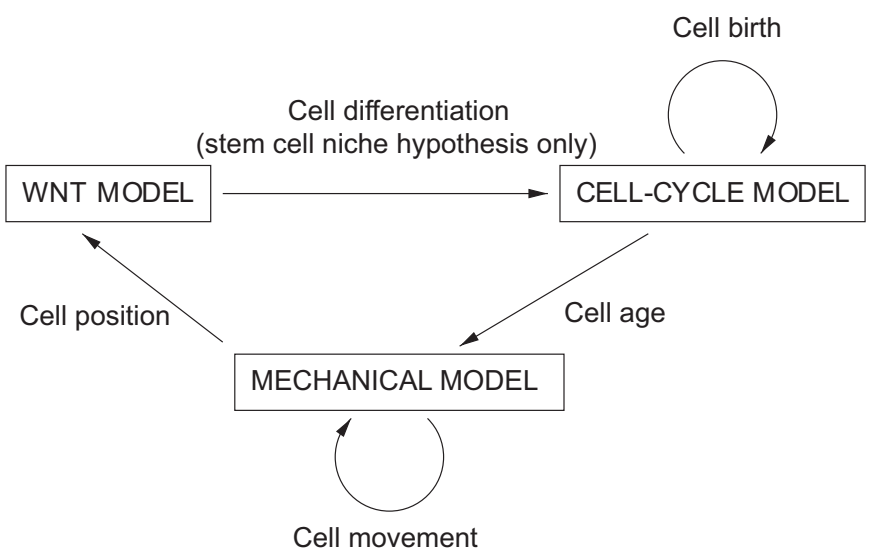

Fig. 2. Diagram illustrating the multiscale nature of the spatial crypt model. At each time step the mechanical model is used to update the position of each cell in the crypt. Cell birth is governed by a cell cycle model. In the case of the stem cell niche hypothesis, this is determined by the local Wnt concentration, which is in turn determined by the location up the crypt. 
suitably modified for a non-flat geometry. There are two basic components to the model: the first step is to decide which cells are neighbors of each other, and the second is to determine the forces transmitted by these neighbors. Each cell $i$ is represented by a point $\mathbf{r}_{i} \in \mathbb{R}^{3}$, which may be interpreted as the center of the nucleus. Cells are constrained to lie on the crypt surface. In Meineke et al. (2001) and van Leeuwen et al. (2009) this is taken to be a circular cylinder. While this assumption is fine for most of the crypt, it is a poor approximation of the base of the crypt where the stem cells reside. Since accurately modeling the stem cells is crucial to our study of monoclonal conversion, here we take better account of the geometry by modeling the surface of the crypt by the surface of revolution

$\Sigma=\left\{(r, \theta, z) \in \mathbb{R}^{3}: z=f(r)\right\}$.

We take $f(0)=0$ so that the bottom of the crypt is located at the origin.

The area on this surface occupied by each cell is determined by a Voronoi tessellation centered on the points $\mathbf{r}_{i}$, that is, the area occupied by the cell associated with $\mathbf{r}_{i}$ is the set of points which are closer to $\mathbf{r}_{i}$ than to any other $\mathbf{r}_{j}, j \neq i$. Strictly speaking we should determine these distances using a non-Euclidean norm to account for the curvature of the surface. However, this is overly complicated given the simple nature of the mechanical model itself, and we determine the cells simply by tessellating the projection in the plane $z=0$. This tessellation tells us which cells are neighbors, and thus which cells can transmit force to a given cell. If we join the centers of neighboring cells then this generates a triangulation of the $\mathbf{r}_{i}$ known as a Delaunay triangulation.

\subsubsection{Cell dynamics}

We assume that cell movement within the crypt is driven by mitotic activity. New-born cells force nearby cells to move away, causing a pressure-driven passive movement primarily up the crypt axis. Following Meineke et al. (2001), we model the local repelling and attracting intercellular forces by a network of springs connecting neighboring cells. The total force $\mathbf{F}_{i}(t)$ acting on a cell $i$ at time $t$ is equal to the sum of all forces coming from the springs of all neighboring cells $j \in \mathcal{N}_{i}(t)$ adjacent to $i$ at that time

$\mathbf{F}_{i}(t)=\mu \sum_{j \in \mathcal{N}_{i}(t)} \hat{\mathbf{r}}_{i j}(t)\left(\left\|\mathbf{r}_{i j}(t)\right\|-s_{i j}(t)\right)$,

where $\mu$ is the spring constant, $\mathbf{r}_{i j}(t)=\mathbf{r}_{i}(t)-\mathbf{r}_{j}(t)$ is the vector from $i$ to $j$ at time $t, \hat{\mathbf{r}}_{i j}(t)$ is the corresponding unit vector and $s_{i j}$ is the natural separation (spring length) between cells $i$ and $j$. Here we are making a local tangent plane approximation in using the distance between two cells in three dimensions rather than in the crypt surface to calculate the force. Again, this is valid so long as the cell diameter is small compared to the radius of curvature of the crypt surface. ${ }^{1}$

Since we are constraining all cells to lie on the crypt surface, we can determine the position of each cell by its projection in the plane $z=0$. The constraint that $\mathbf{r}_{j}$ must lie on $\Sigma$ manifests itself as a reaction force $\mathbf{R}_{j}$ acting normal to the surface whose magnitude balances exactly the normal component of $\mathbf{F}_{i}$. Thus to update the positions of the cells we need the $x$-and $y$-components of the tangential component of $\mathbf{F}_{i}$. These are given by

$\mathbf{F}_{i}^{*}=\mathbf{F}_{i}-\left(\mathbf{F}_{i} \cdot \mathbf{n}_{i}\right) \mathbf{n}_{i}-\left(\mathbf{F}_{i} \cdot \mathbf{e}_{z}\right) \mathbf{e}_{z}+\left(\mathbf{F}_{i} \cdot \mathbf{n}_{i}\right)\left(\mathbf{n}_{i} \cdot \mathbf{e}_{z}\right) \mathbf{e}_{z}$,

where $\mathbf{n}_{i}$ is the unit outward normal to the crypt surface at the point $\mathbf{r}_{i}$, and $\mathbf{e}_{z}$ is the unit vector in the $z$-direction.

\footnotetext{
${ }^{1}$ If this is not the case then treating the cells as two dimensional is itself questionable.
}

We make the standard off-lattice model assumption that the motion of cells is damped (Meineke et al., 2001). This simplifying assumption that inertial terms are small compared to dissipative terms, leads to first-order dynamics with the evolution of the projection $\mathbf{x}_{i}$ for each cell determined by

$\frac{\mathrm{d} \mathbf{x}_{i}}{\mathrm{~d} t}=\frac{1}{\eta} \mathbf{F}_{i}^{*}(t)$

where $\eta$ is the damping constant, which we assume to take the same value for each cell. We solve this equation numerically by using a simple forward Euler discretization, so that the effective displacement within a small time interval $\Delta t$ is given by

$\mathbf{x}_{i}(t+\Delta t)=\mathbf{x}_{i}(t)+\frac{1}{\eta} \mathbf{F}_{i}^{*}(t) \Delta t$.

As this numerical method is explicit, we must choose a sufficiently small time step. If $\Delta t$ is too large, then the numerical solution may exhibit spurious oscillations.

After any cell movement the Delaunay neighborhoods may have changed, so the set of points corresponding to cell centers must be remeshed by a Delaunay triangulation at every time step. The Delaunay triangulation is implemented using a highly optimized, freely available meshing program, ${ }^{2}$ which requires a convex hull. To accommodate this requirement, we extend the crypt projection mesh by adding a surrounding layer of 'ghost' nodes, as shown in Fig. 3; for further details, see van Leeuwen et al. (2009). These nodes do not correspond to real cells and do not exert any forces on those that do. Their purpose is simply to facilitate remeshing and to provide extra boundary points so that the Voronoi tessellation does not result in extremely large cells at the boundary of the mesh (corresponding to the top of the crypt).

\subsubsection{Cell birth}

Cell division is implemented in the model as follows. When the cell cycle model (to be discussed shortly) signals that the associated cell is ready to divide, a new node is added to the mesh a short distance $\varepsilon$ away from the node in the mesh corresponding to the cell, at a random angle. A new cell cycle model and associated cell are created to correspond to this new node. To account for cell growth, and to avoid an unrealistically large force between these two neighboring nodes, we let the rest length of the spring between them increase linearly from $\varepsilon$ to its normal value $\ell$ over the first hour after division

$s_{i j}(t)= \begin{cases}\varepsilon+(\ell-\varepsilon)\left(t-t_{d}\right), & t \leq t_{d}+1, \\ \ell, & t>t_{d}+1,\end{cases}$

where $t_{d}$ is the time at which the parent cell divides.

\subsubsection{Cell sloughing}

It is known that cells that have migrated to the top of the crypt are sloughed off into the lumen. This is implemented in the model as follows. When a node moves beyond a critical radius $R$ from the center of the projected mesh, it is removed from the mesh and the cell corresponding to it is deleted.

\subsection{Cell cycle models}

The cell cycle is the orderly sequence of events by which a eukaryotic cell grows, duplicates its contents and divides into two (Alberts et al., 2002). The cell cycle may be divided into four distinct phases: gap 1 (G1), synthesis (S), gap 2 (G2) and mitosis (M). During G1 phase, the cell grows in volume and monitors its environment. When the external conditions and the size of the

\footnotetext{
${ }^{2}$ Triangle (www.cs.cmu.edu/ quake/triangle.html).
} 

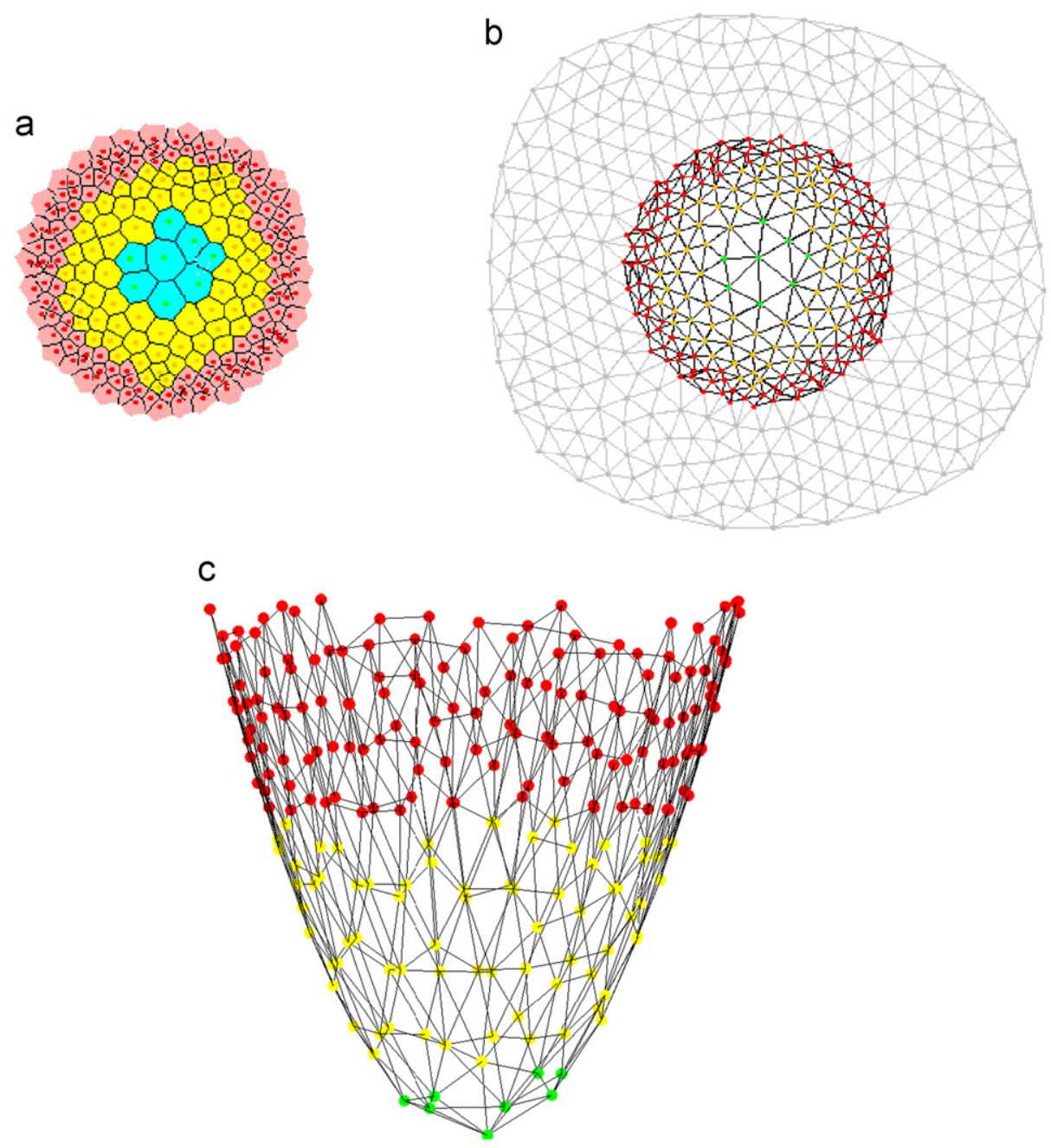

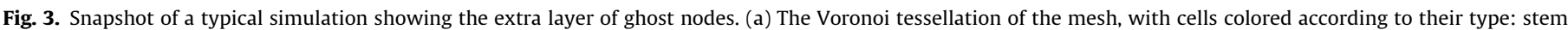

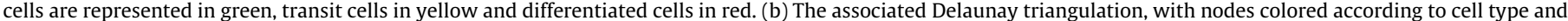

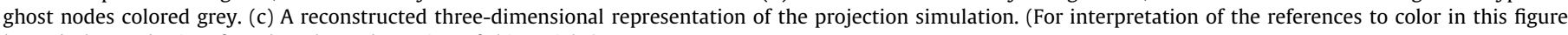
legend, the reader is referred to the web version of this article.)

cell are suitable, the cell passes through a checkpoint called the $\mathrm{G} 1 / \mathrm{S}$ restriction point and enters $\mathrm{S}$ phase, committing itself to DNA replication and division. This transition is irreversible: once the cell enters the $S$ phase and DNA replication commences, division has to be completed. After replicating its DNA, the cell then progresses through G2 phase, which allows additional time for growth. The cell then reaches the G2 checkpoint, and checks that DNA and chromatide alignment have occurred. The cell then enters $M$ phase, during which chromosome segregation and cell division occur.

A number of mathematical models of the cell cycle have been developed, which are usually formulated as systems of coupled nonlinear ordinary differential equations that describe the change in the levels of key cell cycle control proteins (Tyson et al., 2001, 2002). In these models, the passage through cell cycle checkpoints occurs when the levels of specific proteins satisfy threshold conditions. Since these models contain a large amount of biological detail that is not necessary for our purposes, we instead use a much simplified model, in which the durations $t_{S}, t_{G 2}, t_{M}$ of $\mathrm{S}$ phase, G2 phase and $M$ phase, respectively, are fixed, and all stochastic variation (required to break cell division synchrony) is incorporated into the duration of the $\mathrm{G} 1$ phase $t_{G 1}$. The precise nature of this random variable is informed by which stem cell hypothesis we choose.

\subsubsection{Immortal stem cell hypothesis}

To investigate the immortal stem cell hypothesis, the duration of a proliferating cell's $G 1$ phase, $t_{G 1}$, is taken to be a normal random variable with constant variance $\sigma^{2}$ and constant mean $\mu_{\text {stem }}$ or $\mu_{\text {transit }}$ depending on the cell type.

We assume that each stem cell divides asymmetrically to one stem cell and one transit cell with probability $1-\alpha$, and divides symmetrically to either two stem cells or two transit cells, each with probability $\alpha / 2$. It is known that the total number of stem cells in a crypt remains constant on average (Kim and Shibata, 2002; Brittan and Wright, 2002). A necessary condition for this model of stem cell division to exhibit such behavior is that the two symmetric division probabilities must be equal, since otherwise the average number of stem cells would either increase without bound or decrease to zero over time. We therefore consider only this special case of matched symmetric division probabilities. Following Meineke et al. (2001) and Loeffler et al. (1986), we assume that each cell has a pedigree, or generation. Each stem cell is fixed to be of generation zero at division, while the generation of a transit cell is incremented by one at each division. We assume that each transit cell may undergo a certain number of divisions, reaching a maximum generation $g_{\max }$ at which it becomes a differentiated cell that does not proliferate. In their cylindrical crypt model, Meineke et al. (2001) show that 
this mechanism can account for the correct numbers of stem, transit and differentiated cells in a murine intestinal crypt when $g_{\max }=3$.

In contrast to Meineke et al. (2001), we do not fix the positions of stem cells at the bottom of the crypt: this would result in cell crowding, as daughter cells that are placed randomly nearby stem cells may become trapped. Instead it makes sense to assume that stem cells respond to an external chemical cue (in our case, Wnt) by active migration. This chemotactic behavior ensures that, although motile, stem cells tend to move down towards the bottom of the crypt if displaced as a result of mitosis. This is implemented as an additional force in the equation of motion (1) for each stem cell

$\mathbf{F}_{i}(t)=\mu \sum_{j \in \mathcal{N}_{i}(t)} \hat{\mathbf{r}}_{i j}(t)\left(\left\|\mathbf{r}_{i j}(t)\right\|-s_{i j}(t)\right)+\chi \nabla W\left(\mathbf{r}_{i}(t)\right)$,

where the Wnt concentration $W(\mathbf{r})$ is prescribed to be linear and constant in time, and the strength of the chemotactic force $\chi$ is chosen to ensure that the equilibrium configuration of stem cells is not too closely packed. We emphasize here that although we have assumed that Wnt plays the role of a chemical cue, the precise nature or mechanism of this cue is not critical to the model: some form of graded signal is all that is assumed.

An alternative modeling assumption would be that stem cells are pulled back down the crypt as a result of substrate forces. However, this raises the question of how cells in the basal lamina communicate with the stem cells and pull them in the right direction. The simplest explanation for this is the presence of an external chemical cue, which brings us back to our original assumption.

\subsubsection{Stem cell niche hypothesis}

Under the stem cell niche hypothesis, we assume that a cell has no intrinsic 'stemness' but performs the role of a stem cell if it is born in the presence of a relatively high Wnt concentration. In line with recent experimental evidence (Gaspar and Fodde, 2004; van de Wetering et al., 2002), we superimpose a spatial gradient of Wnt along the vertical crypt axis, with high levels of Wnt at the crypt base and low levels at the lumen. We incorporate into each cell a
Wnt-dependent cell-cycle model which leads to position-dependent cell-cycle times. All division is assumed to be symmetric. A cell divides symmetrically into two stem cells at high Wnt levels and two transit cells at lower Wnt levels. If the Wnt level is lower still, then the cell does not divide. Hence cell type is a result of the current local Wnt level. As in the immortal stem cell hypothesis, the duration of a proliferating cell's $G 1$ phase, $t_{G 1}$, is given by a normal random variable with cell-type dependent means $\mu_{\text {stem }}, \mu_{\text {transit }}$ and constant variance $\sigma^{2}$. Differentiated cells are assumed to have $t_{G 1}=\infty$. We shall usually take $\mu_{\text {transit }}=\mu_{\text {stem }}$ under this hypothesis, in accordance with the notion that stem cells differ from transit cells purely in their location in the crypt; although we will also consider the case where $\mu_{\text {transit }} \neq \mu_{\text {stem }}$ for comparison.

The Wnt gradient is imposed to replicate the expected positions of stem, transit and differentiated cells in the crypt. We superimpose a linear Wnt gradient of the form

$W(r)=W_{0}(R-r)$

where $W_{0}$ denotes the Wnt concentration at the base of the crypt and $R$ denotes the radius of the crypt at its apex. We then define two critical Wnt concentrations: $W_{\text {stem }}$, the concentration above which new cells may be considered to be stem cells, and $W_{\text {transit }}$, the concentration below which cells may be considering to be terminally differentiated.

\subsection{Parameter values}

In this section we list the parameter values used in the crypt model. In all simulations we have set $\Delta t=30 \mathrm{~s}$, and have established through numerical experiments that this value is small enough to confer stability on the numerical method. All lengths are scaled with the spring rest length $\ell$ between neighboring cells, which is approximately $10 \mu \mathrm{m}$ (Drasdo and Loeffler, 2001). We approximate the surface of the crypt by the paraboloid $z=a r^{2}$. The value of $a$ is guided by Halm and Halm (2000), who obtain the average height of a human colonic crypt as $433 \mu \mathrm{m}$ and its the width at the top of the crypt as $74 \mu \mathrm{m}$. Here we assume that murine and human crypts are similar (in the geometrical sense). The value of the sloughing radius $R$ is chosen to ensure that the average
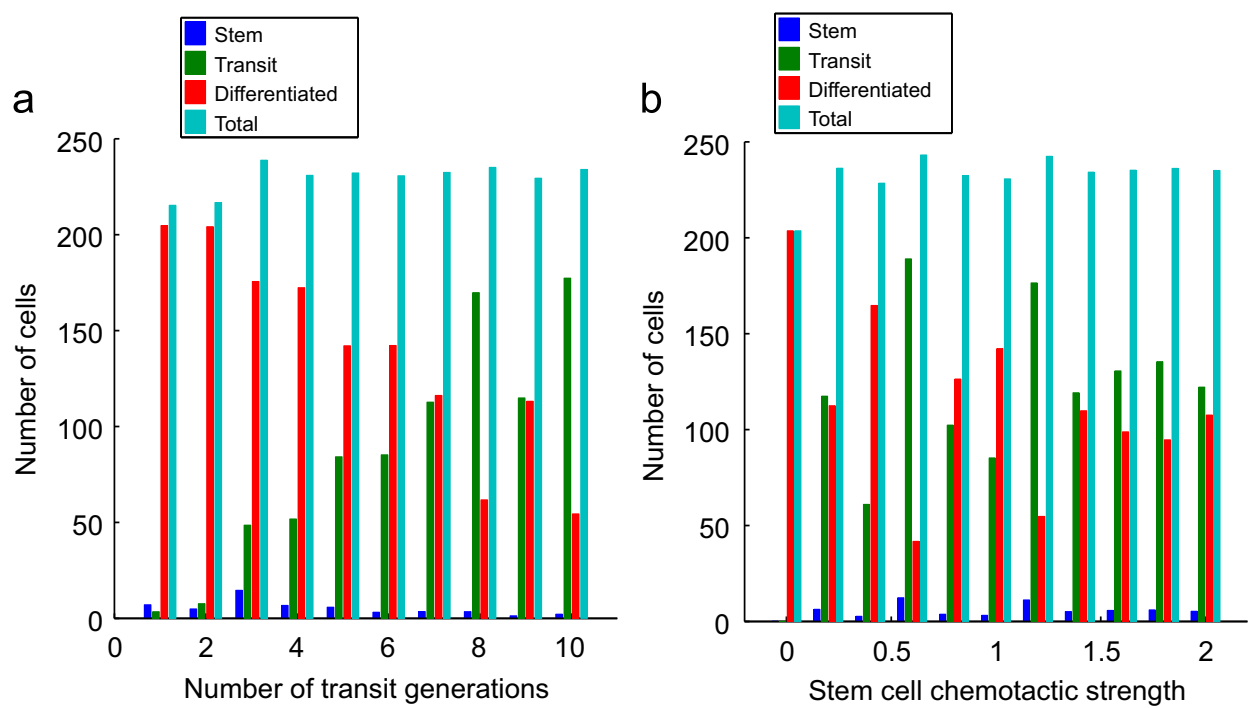

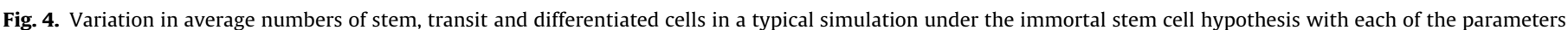

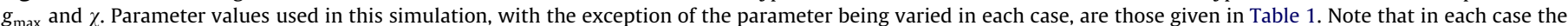

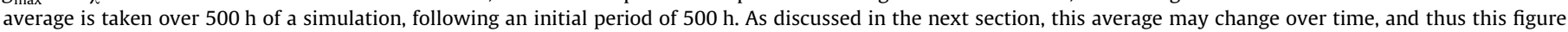

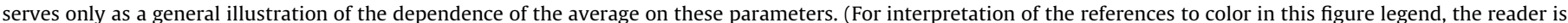
referred to the web version of this article.) 
Table 1

Parameter values for the crypt model. Estimation of parameter values for which no reference is given is discussed in the main text.

\begin{tabular}{llll}
\hline Parameter & Description & Estimate & Reference \\
\hline$a$ & Shape parameter & 3 & Halm and Halm (2000) \\
$R$ & Crypt height & 5 & - \\
$\eta$ & Damping constant & 1 & Meineke et al. (2001) \\
$\mu$ & Spring constant & 15 & Meineke et al. (2001) \\
$\varepsilon$ & Mitotic spring length & 0.5 & Meineke et al. (2001) \\
$\ell$ & Natural spring length & 1 & - \\
$\Delta t$ & Time step & $0.0083 \mathrm{~h}$ & - \\
$\mu_{\text {stem }}$ & Mean stem cell G1 duration & $14 \mathrm{~h}$ & Meineke et al. (2001) \\
$\mu_{\text {transit }}$ & Mean transit cell G1 duration & $2 \mathrm{~h}$ & Meineke et al. (2001) \\
$\sigma^{2}$ & G1 duration variance & $1 \mathrm{~h}$ & Meineke et al. (2001) \\
$t_{S}$ & S phase duration & $5 \mathrm{~h}$ & Meineke et al. (2001) \\
$t_{G 2}$ & G2 phase duration & $4 \mathrm{~h}$ & Meineke et al. (2001) \\
$t_{M}$ & M phase duration & $1 \mathrm{~h}$ & Meineke et al. (2001) \\
$\alpha$ & Probability of symmetric division & 0.1 & - \\
$\chi$ & Stem cell chemotactic strength & 1 & - \\
$g_{\text {max }}$ & Number of transit generations & 6 & - \\
$W_{0}$ & Basal Wnt concentration & 1 & - \\
$W_{\text {stem }}$ & Stem cell Wnt concentration & 0.8 & - \\
$W_{\text {transit }}$ & Transit cell Wnt concentration & 0.65 & - \\
\hline & & &
\end{tabular}

number of cells in the crypt is approximately 250, as observed experimentally (Potten and Loeffler, 1990).

Under the immortal stem cell hypothesis, a larger number of transit generations than that are given by Meineke et al. (2001) are required to give approximately the correct proportions of transit and differentiated cells in the crypt. This is because the crypt projection geometry allows for cells to move tangentially to the crypt axis more easily than in the cylindrical geometry. Based upon numerical investigations, we choose $g_{\max }=6$. Similarly, we choose a value of the chemotactic strength $\chi$ that results in realistic packing of stem cells at the base of the crypt. Fig. 4 shows how the number of stem, transit and differentiated cells, averaged over $500 \mathrm{~h}$ of a simulation under the immortal stem cell hypothesis, varies with each of these parameters. The probability of symmetric division $\alpha$ must not be too large, or else the number of stem cells in the crypt will rapidly decrease to zero (see Results section). Under the stem cell niche hypothesis, the Wnt concentration is scaled with its value $W_{0}$ at the base of the crypt. The critical Wnt concentrations $W_{\text {stem }}$ and $W_{\text {transit }}$ are chosen to ensure that the average number of 'stem' cells in the crypt is approximately 8 (Boman et al., 2001; Marshman et al., 2002; Potten and Loeffler, 1990) and the average number of proliferating cells in the crypt is approximately 150 (Potten and Loeffler, 1990). All other parameter values are listed with references in Table 1.

\subsection{Model implementation}

Model simulations were implemented within the computational framework of the Chaste project (Pitt-Francis et al., 2009).

\section{Results}

\subsection{Immortal stem cell hypothesis}

Simulations of the crypt model under the immortal stem cell hypothesis showed that even with a small probability of symmetric division, the crypt eventually loses all its stem cells, an event that is not biologically realistic (a typical model simulation under the immortal stem cell hypothesis is shown in Fig. 5). Furthermore, even while the number of stem cells varied slowly, the numbers of transit cells and differentiated cells oscillated wildly, as shown in Fig. 6. We therefore conclude the immortal stem cell hypothesis alone cannot explain the observed crypt structure. Indeed, under this hypothesis, each clonal population will (given sufficient time) become extinct. Thus every crypt will, eventually, lose all its stem cells. This process is referred to as crypt extinction.

Although such crypt extinction events have been observed experimentally, they are extremely rare, and usually the total number of stem cells in a crypt remains constant on average (Kim and Shibata, 2002; Brittan and Wright, 2002). This is an inherent weakness of the immortal stem cell hypothesis as formulated in our model. It means that some type of population-dependent feedback is required to allow for the replenishment of stem cells. This issue has been addressed to some extent using non-spatial modeling, with several forms of feedback considered, most notably de-differentiation of transit cells (Johnston et al., 2007), which may give realistic behavior at the cell population level. However, when one considers spatial effects, a problem is encountered. De-differentiation must either be a random process, or a result of spatially varying cues. Under the former hypothesis, one would expect to observe transit cells de-differentiating at random locations up the crypt, but this is not observed experimentally. Under the latter hypothesis, we may recover the typical spatial crypt structure, but this is in effect a form of the stem cell niche hypothesis. We therefore proceed to study the model behavior under this hypothesis.

\subsection{Niche hypothesis}

In our model, the stem cell niche hypothesis naturally leads to a stable crypt structure with the requisite numbers of stem, transit and differentiated cells. Figs. 7-9 show the results of a typical crypt simulation, in which each cell in the crypt is initially labeled with a different clonal index, and their progeny tracked over time until the crypt becomes monoclonal. As Fig. 7 demonstrates, the numbers of stem cells, transit cells and differentiated cells remain constant on average, and are much more stable than under the immortal stem cell hypothesis.

Under the niche hypothesis, monoclonal conversion is inevitable as, over time, the progeny of one clonal population take over the entire stem cell niche, and then soon after the entire crypt. As Figs. 8 and 9 demonstrate, there is a fast initial drop in the number of different clonal populations in the first few days, as non-proliferating cells are swept out of the crypt. This is typically followed by a longer period during which two or more clonal populations compete 


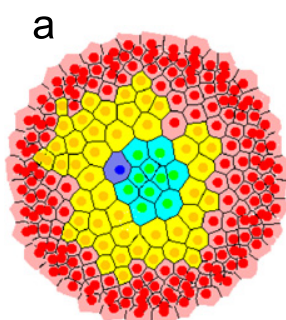

$t=0 \mathrm{~h}$

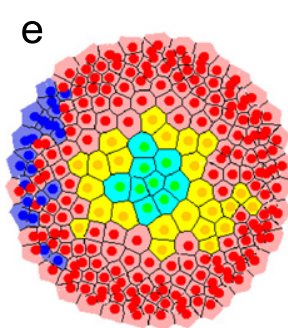

$t=120 \mathrm{~h}$

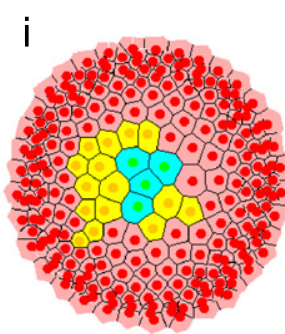

$t=240 \mathrm{~h}$

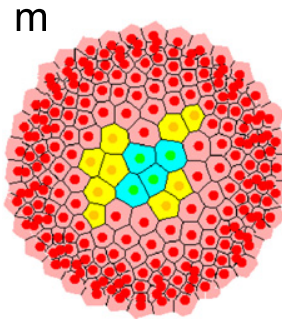

$t=360 \mathrm{~h}$

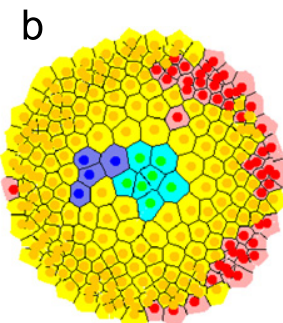

$t=30 \mathrm{~h}$

$\mathrm{f}$
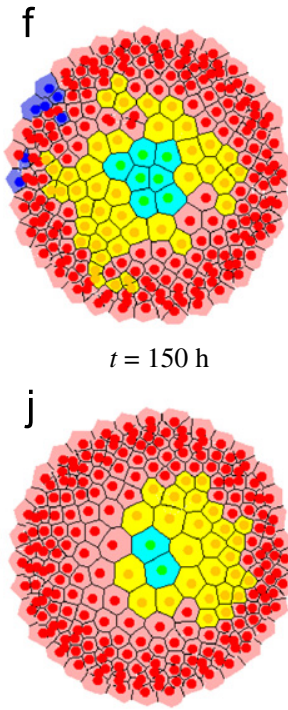

$t=270 \mathrm{~h}$

$\mathrm{n}$

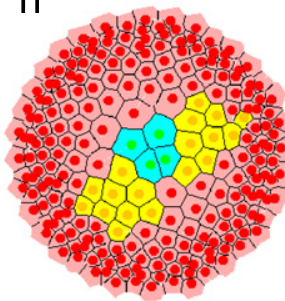

$t=390 \mathrm{~h}$

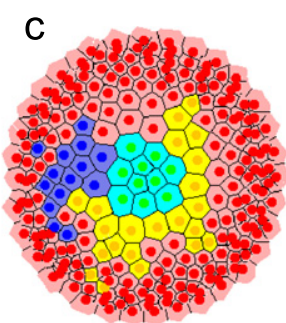

$t=60 \mathrm{~h}$

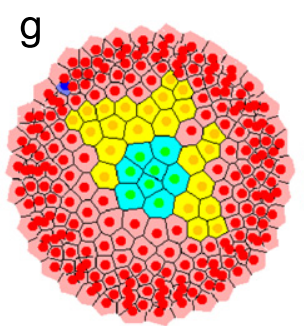

$=180 \mathrm{~h}$

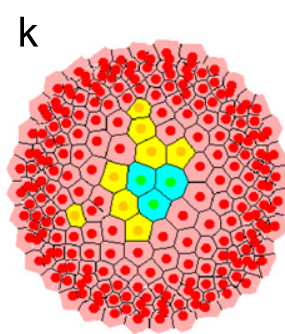

$t=300 \mathrm{~h}$

0

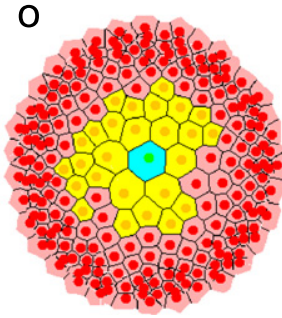

$t=420 \mathrm{~h}$

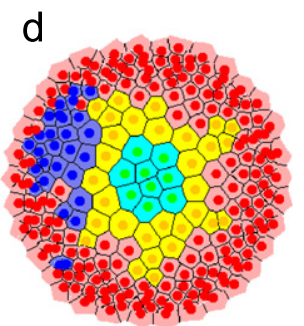

$t=90 \mathrm{~h}$

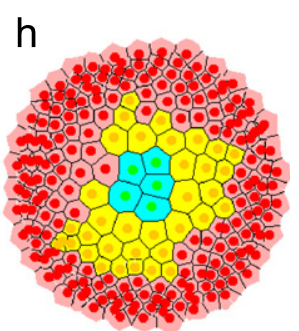

$t=210 \mathrm{~h}$

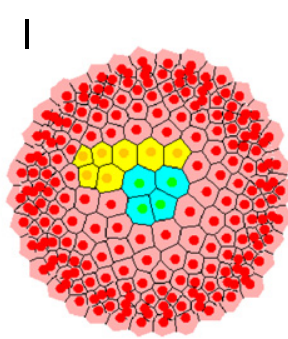

$t=330 \mathrm{~h}$

$\mathrm{p}$

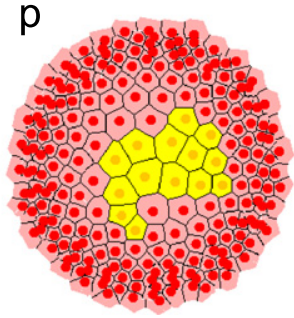

$t=440 \mathrm{~h}$

Fig. 5. Time course showing stem cell extinction in the colonic crypt under the immortal stem cell hypothesis. Stem cells are represented in green, transit cells in yellow and differentiated cells in red. Parameter values used in this simulation are those given in Table 1 with $\mu_{\text {stem }}=\mu_{\text {transit }}=2 \mathrm{~h}$. Initially a single cell in the stem cell niche is given a harmless mutation (blue) which is transmitted to the cell's progeny. After $180 \mathrm{~h}$ this clonal population has been swept out off the crypt (g). There is significant variation in the numbers of spatial distribution of different cell types, and after $440 \mathrm{~h}$ the number of stem cells present has dropped to zero (p). (For interpretation of the references to color in this figure legend, the reader is referred to the web version of this article.)

to take over the niche. This competition is also illustrated by Fig. 10, which shows the results of another simulation in which two clonal populations persist for several weeks, exhibiting oscillations in cell numbers, until one clonal population is eventually finally swept out of the niche and the crypt becomes monoclonal.

Fig. 11 shows snapshots of a simulation in which a single cell is labeled with a harmless mutation and its progeny tracked, until the crypt has become monoclonal. Of course, to produce these results, we tracked the progeny of all initial cells, as each stem cell in the niche has probability $1 / n$ of becoming the dominant one, where $n$ is the number of cells in the niche.

\subsubsection{Distribution of monoclonal conversion times under niche hypothesis}

We ran 500 simulations of the crypt model under the stem cell niche hypothesis, with equal mean cell cycle times for all proliferating cells $\left(\mu_{\text {stem }}=\mu_{\text {transit }}=2 \mathrm{~h}\right.$ ), and for each simulation recorded the time taken to achieve monoclonality. The results are shown in red in Figs. 12 and 13. The monoclonal conversion times have a $\Gamma$-like distribution, with mean 18.6 days and variance 82.9 days.

We also performed simulations in which stem cells had different mean cell cycle times $\left(\mu_{\text {stem }} \neq \mu_{\text {transit }}\right.$, with values given in Table 1$)$, as has been suggested (Reya et al., 2001). The results are shown in blue in Figs. 12 and 13, allowing a comparison to be made with the case of equal mean cell cycle times. In the case of different mean cell cycle times, the mean monoclonal conversion times was approximately doubled to 36.6 days, with variance 489.7 days. This doubling reflects that of the mean stem cell cycle time.

\subsubsection{Comparison of results with cylindrical geometry}

We also performed simulations using the cylindrical geometry employed by Meineke et al. (2001), in which the crypt surface is 


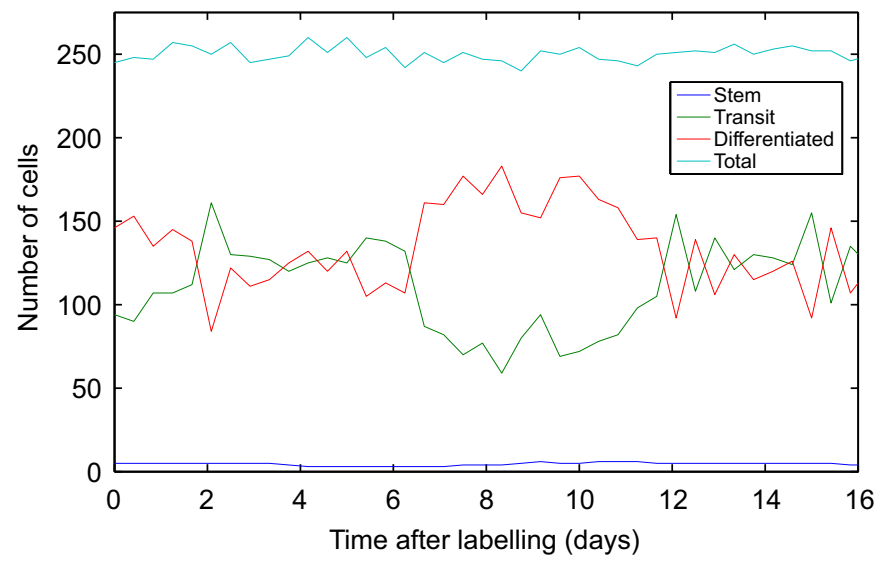

Fig. 6. Numbers of each cell type in a typical simulation under the immortal stem cell hypothesis. Parameter values used in this simulation are those given in Table 1 with $\mu_{\text {stem }}=\mu_{\text {transit }}=2 \mathrm{~h}$. (For interpretation of the references to color in this figure legend, the reader is referred to the web version of this article.)

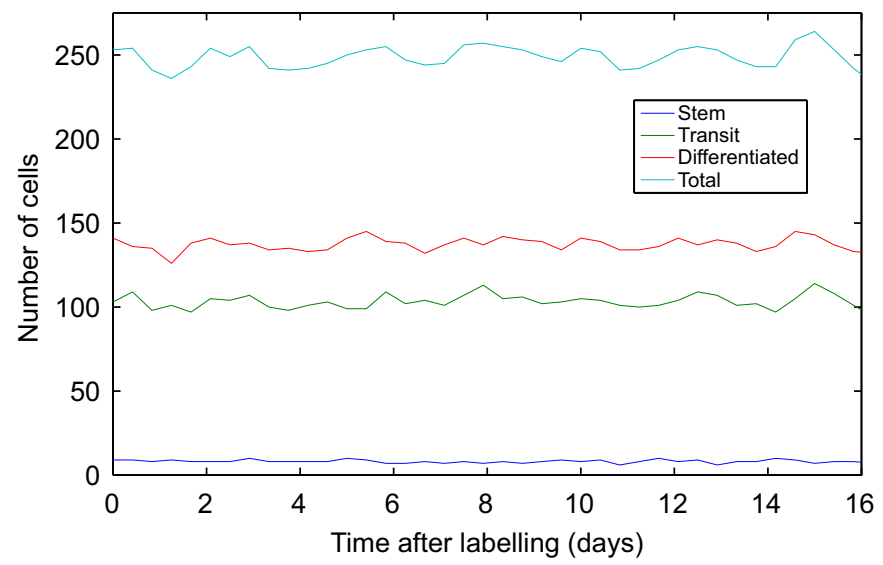

Fig. 7. Numbers of each cell type in a typical crypt simulation under the stem cell niche hypothesis. Parameter values used in this simulation are those given in Table 1 with $\mu_{\text {stem }}=\mu_{\text {transit }}=2 \mathrm{~h}$. (For interpretation of the references to color in this figure legend, the reader is referred to the web version of this article.)

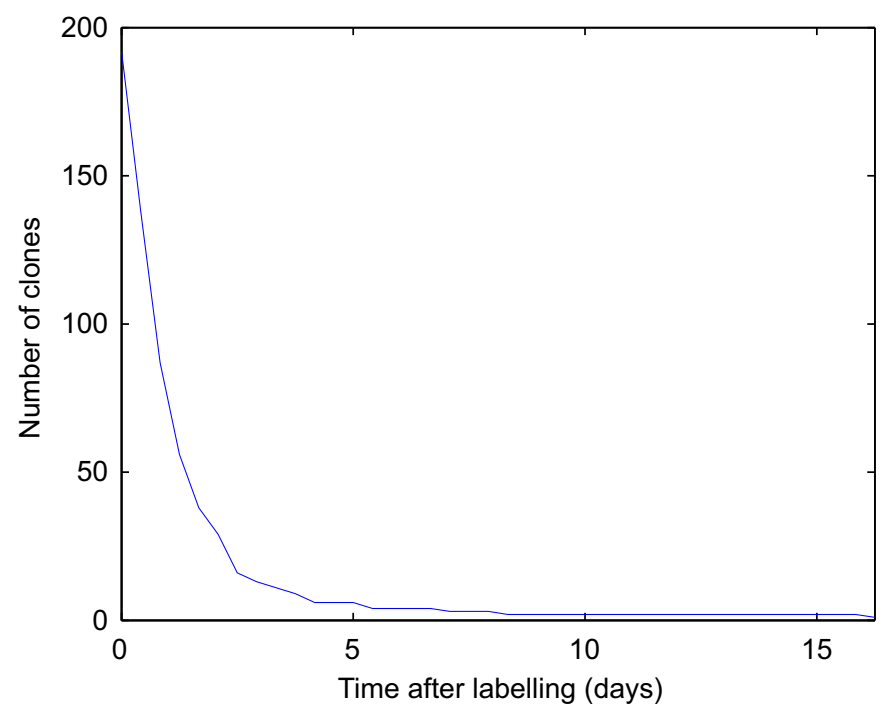

Fig. 8. Number of clonal populations in a typical crypt simulation under the stem cell niche hypothesis. Parameter values used in this simulation are those given in Table 1 with $\mu_{\text {stem }}=\mu_{\text {transit }}=2 \mathrm{~h}$.

modeled as a cylinder, with the same Wnt-dependent cell cycle model described earlier. Results for the case where the mean cell cycle duration is the same for all proliferating cells $\left(\mu_{\text {stem }}=\mu_{\text {transit }}=\right.$

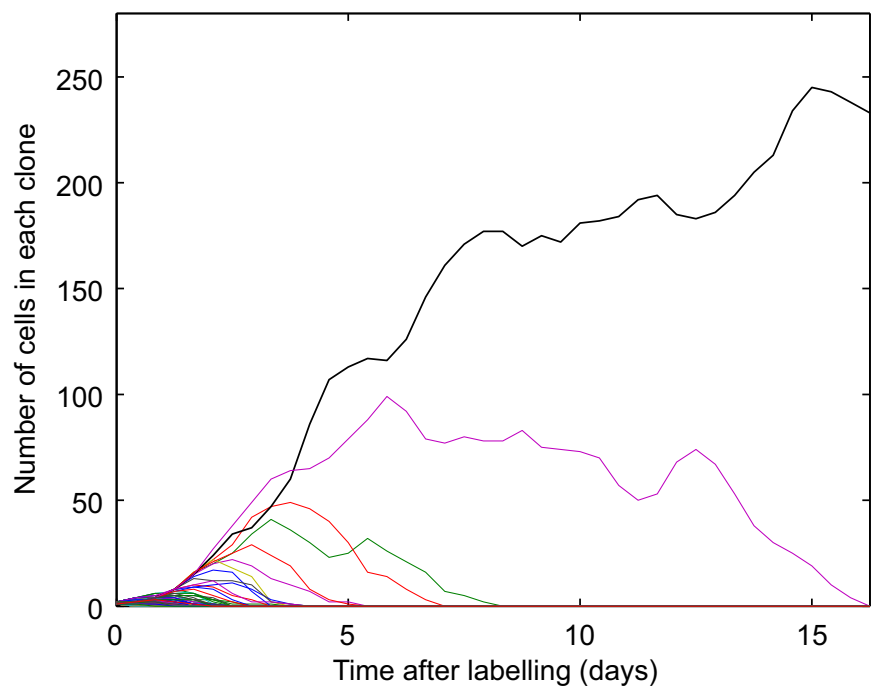

Fig. 9. Number of cells in each clonal population in a typical crypt simulation under the stem cell niche hypothesis. Parameter values used in this simulation are those given in Table 1 with $\mu_{\text {stem }}=\mu_{\text {transit }}=2 \mathrm{~h}$. In this case monoclonal conversion occurs quite quickly.

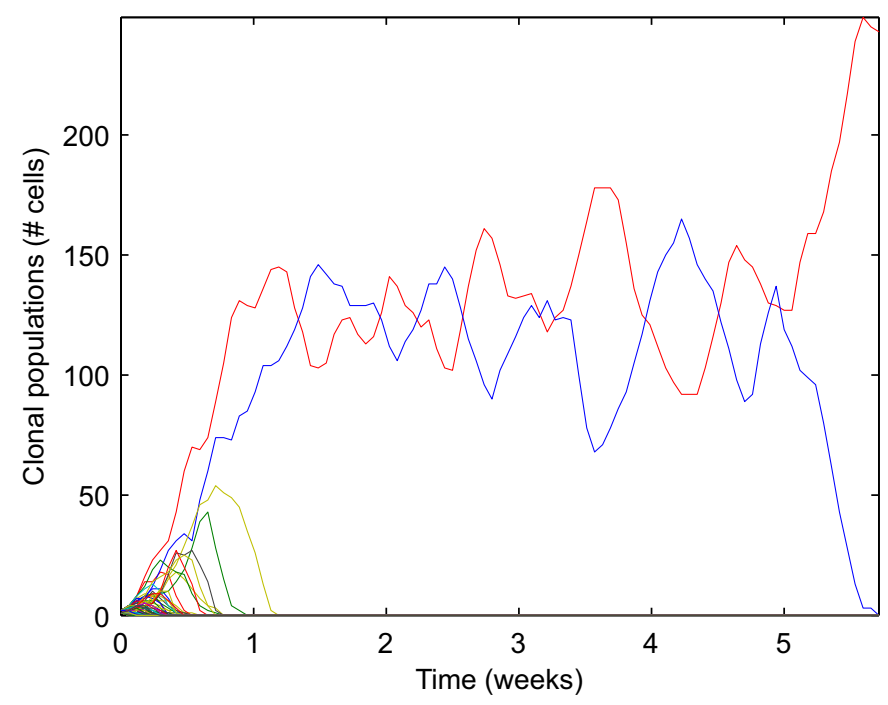

Fig. 10. Number of cells in each clonal population in another crypt simulation under the stem cell niche hypothesis. Parameter values used in this simulation are those given in Table 1 with $\mu_{\text {stem }}=\mu_{\text {transit }}=2 \mathrm{~h}$. In this case monoclonal conversion occurs more slowly, as two clonal populations compete to take over the crypt.

$2 \mathrm{~h}$ ) are shown in Figs. 14 and 15. In this case the mean monoclonal conversion time in this cylindrical model was 42.0 days, significantly higher than when employing the crypt projection geometry, with variance 538.1 days. We also compared the case where cells in the niche have a longer mean G1 duration $\left(\mu_{\text {stem }}=14 \mathrm{~h}, \mu_{\text {transit }}=2 \mathrm{~h}\right)$, results of which are shown in Figs. 16 and 17. In this case the mean monoclonal conversion time using a cylindrical geometry was 58.0 days, with variance 919.1 days. In both cases, the assumption of a simplified cylindrical geometry results in estimated monoclonal conversion times that are significantly higher than those predicted using the crypt projection geometry.

\section{Discussion}

In this section we will seek to understand the results of our multiscale model through simple non-spatial models. We begin in 


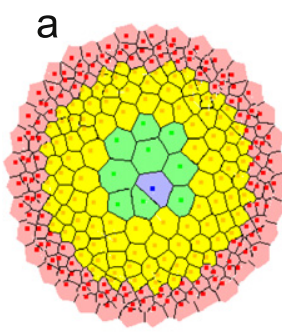

$t=0 \mathrm{~h}$

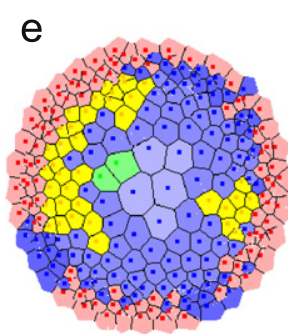

$t=100 \mathrm{~h}$

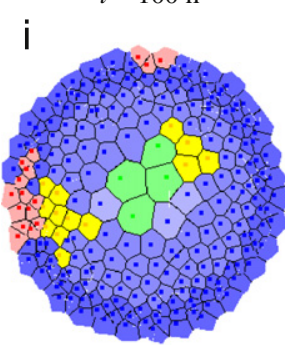

$t=200 \mathrm{~h}$

m

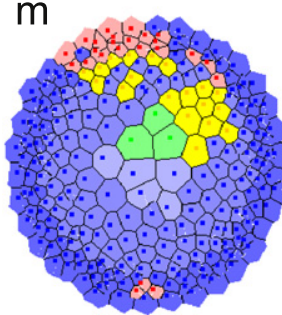

$t=300 \mathrm{~h}$

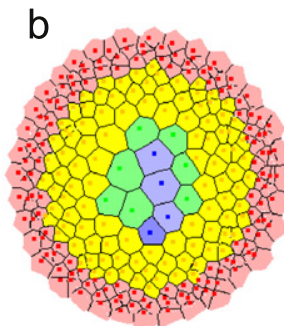

$t=25 \mathrm{~h}$

f

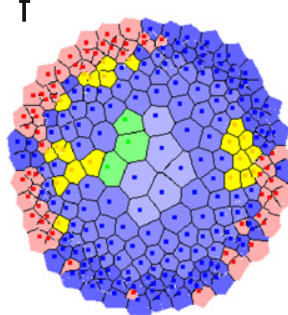

$t=125 \mathrm{~h}$

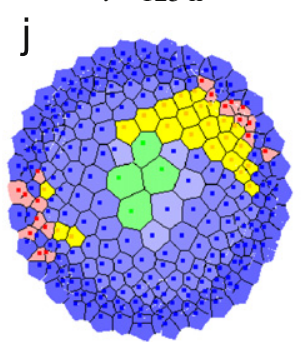

$t=225 \mathrm{~h}$

n

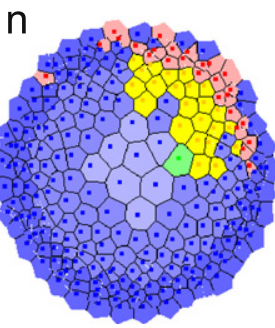

$t=325 \mathrm{~h}$
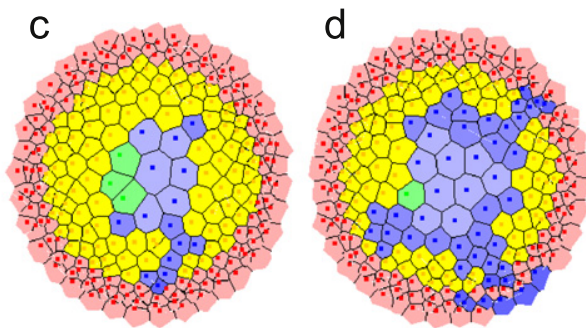

$t=50 \mathrm{~h}$

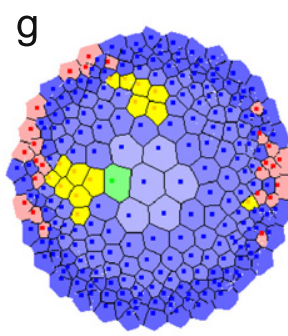

$t=150 \mathrm{~h}$

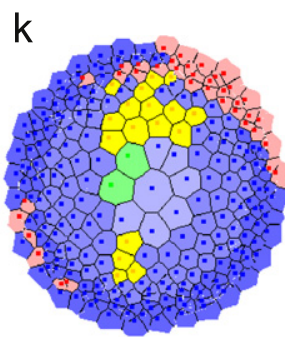

$t=250 \mathrm{~h}$

O

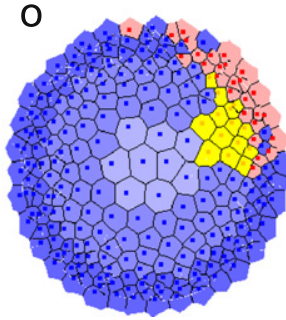

$t=350 \mathrm{~h}$ $t=75 \mathrm{~h}$

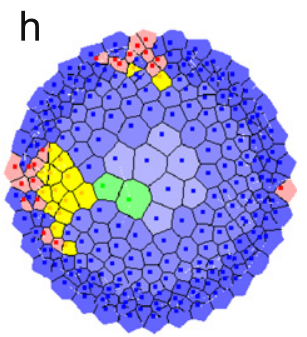

$t=175 \mathrm{~h}$

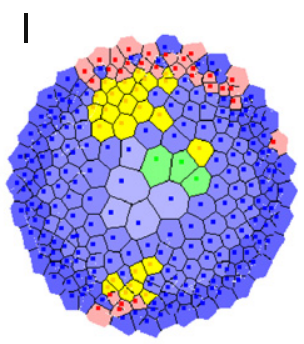

$t=275 \mathrm{~h}$

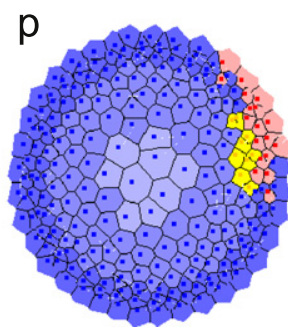

$t=375 \mathrm{~h}$

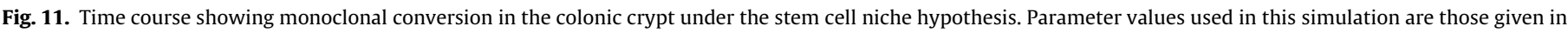

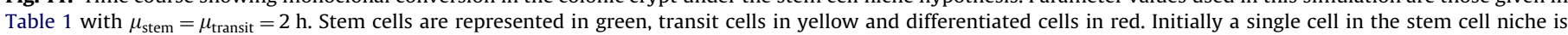

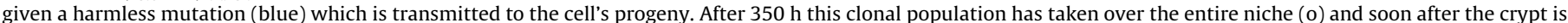

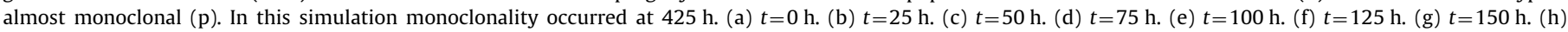

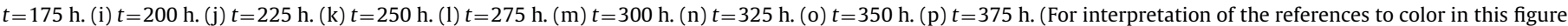
legend, the reader is referred to the web version of this article.)

Sections 4.1 and 4.2 by analyzing two simplified models of stem cell dynamics under the immortal stem cell hypothesis, in which we model stem cell division as a branching process. Then, in Section 4.3, we look at a simplified model of the crypt under the stem cell niche hypothesis, in which the crypt dynamics are modeled as a Moran process.

\subsection{Stochastic model of immortal stem cell hypothesis}

We assume no spatial effects or feedback and consider the population dynamics of a crypt initially containing $n_{0}$ stem cells. Each stem cell may undergo symmetric division to two stem cells (with probability $\alpha / 2$ ), symmetric division to two transit cells (with probability $\alpha / 2$ ) or asymmetric division to one stem cell and one transit cell (with probability $1-\alpha$ ). To incorporate the limited size of the crypt, we place an upper bound $N$ on the size of any given stem cell clone. If this maximum is reached for a given stem cell clone, then at the next division one daughter cell is removed from the crypt; if the division is asymmetric, then the daughter stem cell is removed with probability $\gamma$.

The crypt is considered to be monoclonal when $n_{0}-1$ of the stem cell clones have become extinct (this will actually be a slight underestimate, since it neglects the time taken for the rest of the crypt to be taken over). We begin by studying the dynamics of a single clone, starting with a single stem cell, and compute how many divisions on average are required before extinction occurs.

Let $X_{k} \in\{0, \ldots, N\}$ denote the number of stem cells of a given clone remaining in the crypt after there have been $k$ divisions of this clone. Since the number of stem cells after $k+1$ divisions depends just on the number of stem cells after $k$ divisions, the sequence $\left(X_{k}\right)_{k \geq 0}$ forms a Markov chain, with one-step transition 


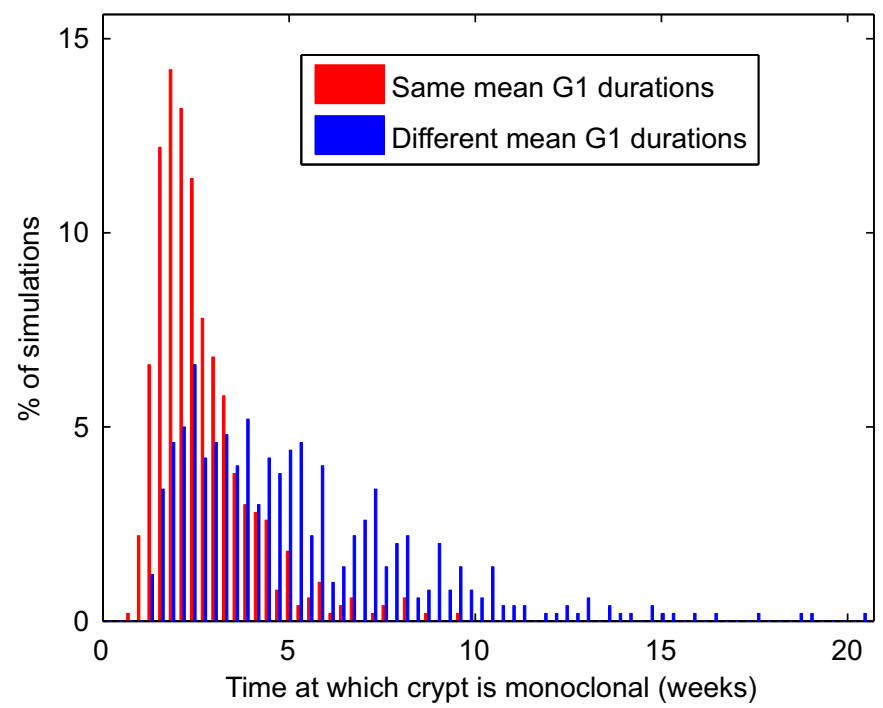

Fig. 12. Comparison of the distribution of monoclonal conversion times for the case where the mean cell cycle duration is the same for all proliferating cells $\left(\mu_{\text {stem }}=\mu_{\text {transit }}=2 \mathrm{~h}\right.$ ) with the case where cells in the niche have a longer mean G1 duration $\left(\mu_{\text {stem }}=14 \mathrm{~h}, \mu_{\text {transit }}=2 \mathrm{~h}\right.$ ). The distributions were obtained by running 500 simulations in each case. (For interpretation of the references to color in this figure legend, the reader is referred to the web version of this article.)

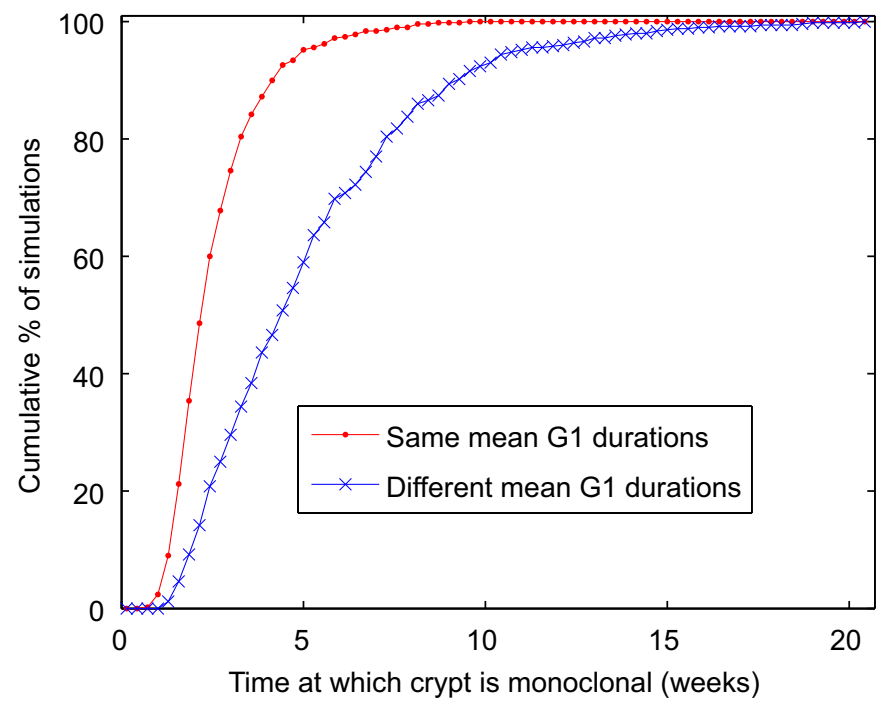

Fig. 13. Comparison of the cumulative distribution of monoclonal conversion times for the case where the mean cell cycle duration is the same for all proliferating cells $\left(\mu_{\text {stem }}=\mu_{\text {transit }}=2 \mathrm{~h}\right)$ with the case where cells in the niche have a longer mean G1 duration $\left(\mu_{\text {stem }}=14 \mathrm{~h}, \mu_{\text {transit }}=2 \mathrm{~h}\right)$. The distributions were obtained by running 500 simulations in each case.

probabilities $p_{i j}=\mathbb{P}\left(X_{k}=j \mid X_{k-1}=i\right)$ given by

$p_{00}=1$,

$p_{i j}=(1-\alpha) \delta_{i, j}+\frac{\alpha}{2} \delta_{i, j \pm 1}, \quad 0<i, j<N$,

$p_{N j}=\left(\frac{\alpha}{2}+\gamma(1-\alpha)\right) \delta_{N-1, j}+\left(\frac{\alpha}{2}+(1-\gamma)(1-\alpha)\right) \delta_{N, j}$,

where $\delta_{i, j}$ denotes the Kronecker delta function

$\delta_{i, j}= \begin{cases}1 & \text { if } i=j, \\ 0 & \text { if } i \neq j .\end{cases}$

This Markov chain is stationary, irreducible and has a single absorbing state $X_{k}=0$, from which it cannot escape. Thus,

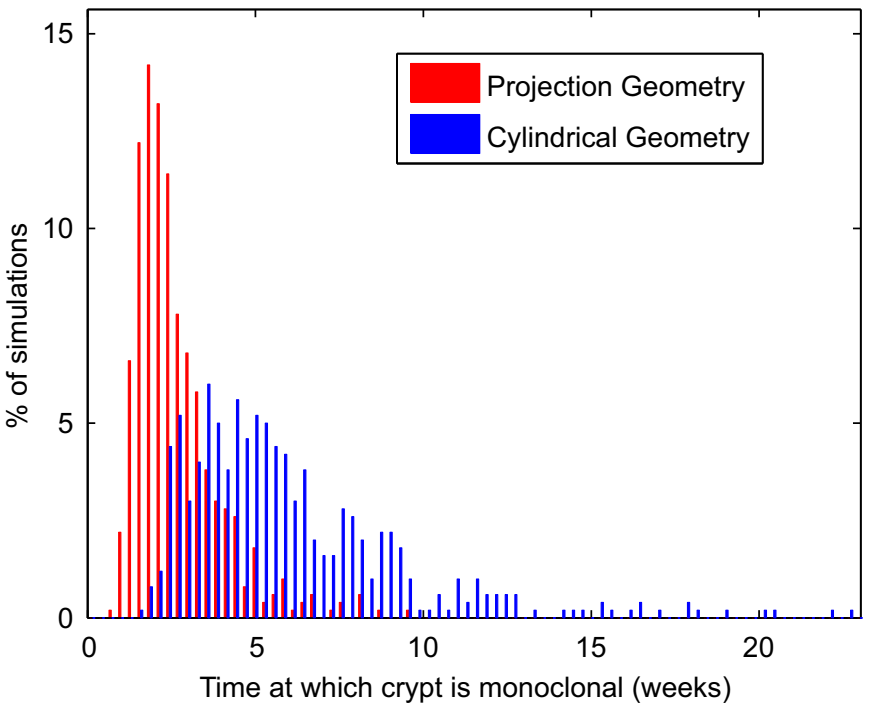

Fig. 14. Comparison of the cumulative distribution of monoclonal conversion times for the cylindrical and projection geometries. The distributions were obtained by running 500 simulations for each geometry. Parameter values used in simulations are those given in Table 1 with $\mu_{\text {stem }}=\mu_{\text {transit }}=2 \mathrm{~h}$. (For interpretation of the references to color in this figure legend, the reader is referred to the web version of this article.)

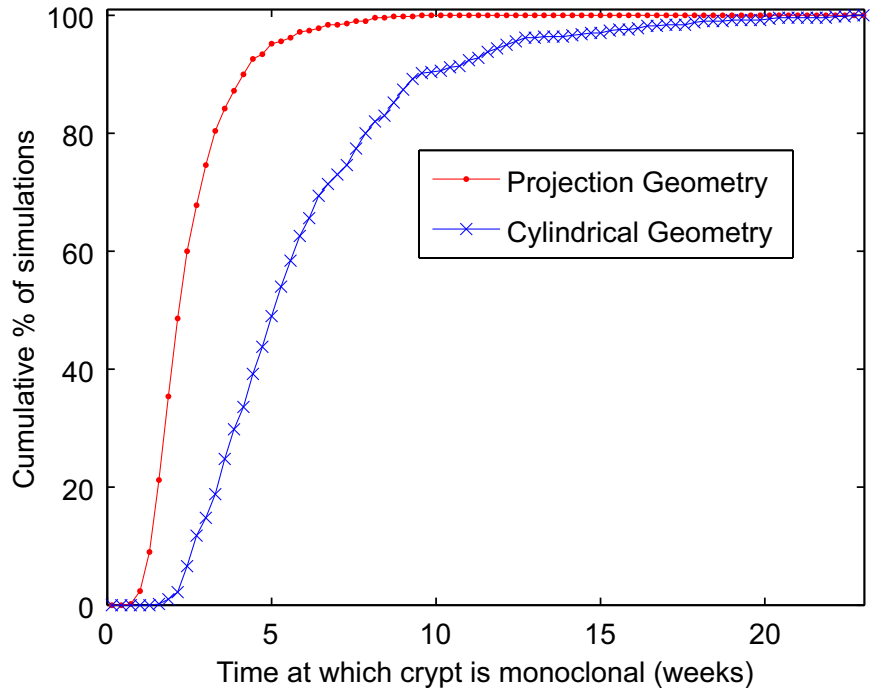

Fig. 15. Comparison of the cumulative distribution of monoclonal conversion times for the cylindrical and projection geometries. The distributions were obtained by running 500 simulations for each geometry. Parameter values used in simulations are those given in Table 1 with $\mu_{\text {stem }}=\mu_{\text {transit }}=2 \mathrm{~h}$.

regardless of the initial number of stem cells, the crypt is doomed to lose all its stem cells in this model. This is analogous to a gambler's ruin problem (Norris, 1997).

The Markov chain $\left(X_{k}\right)_{k \geq 0}$ may behave in one of various qualitatively different ways depending, for instance, on whether or not it ultimately becomes extinct. One way of gaining more insight is to study the behavior of $X_{k}$ conditional upon the occurrence of some event (such as extinction) (Grimmett and Stirzaker, 1992). A natural next step is to ask how long, on average, it takes for extinction to occur. Let $\tau_{i}$ denote the mean number of divisions before first reaching the absorbing state 0 , starting with $X_{0}=i$. It can be shown by conditioning on the first division (Norris, 1997) that $\left(\tau_{0}, \tau_{1}, \ldots, \tau_{N}\right)$ are given by the minimal non-negative solution to the system of 


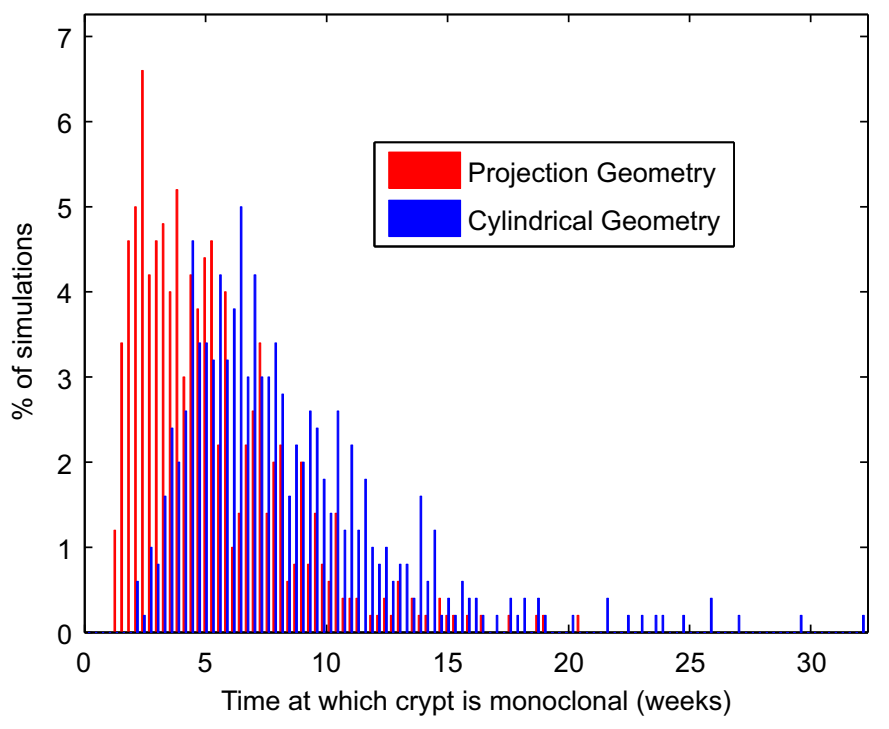

Fig. 16. Comparison of the cumulative distribution of monoclonal conversion times for the cylindrical and projection geometries for the case where cells in the niche have a longer mean G1 duration. The distributions were obtained by running 500 simulations for each geometry. Parameter values used in simulations are those given in Table 1 with $\mu_{\text {stem }}=14 \mathrm{~h}$ and $\mu_{\text {transit }}=2 \mathrm{~h}$. (For interpretation of the references to color in this figure legend, the reader is referred to the web version of this article.)

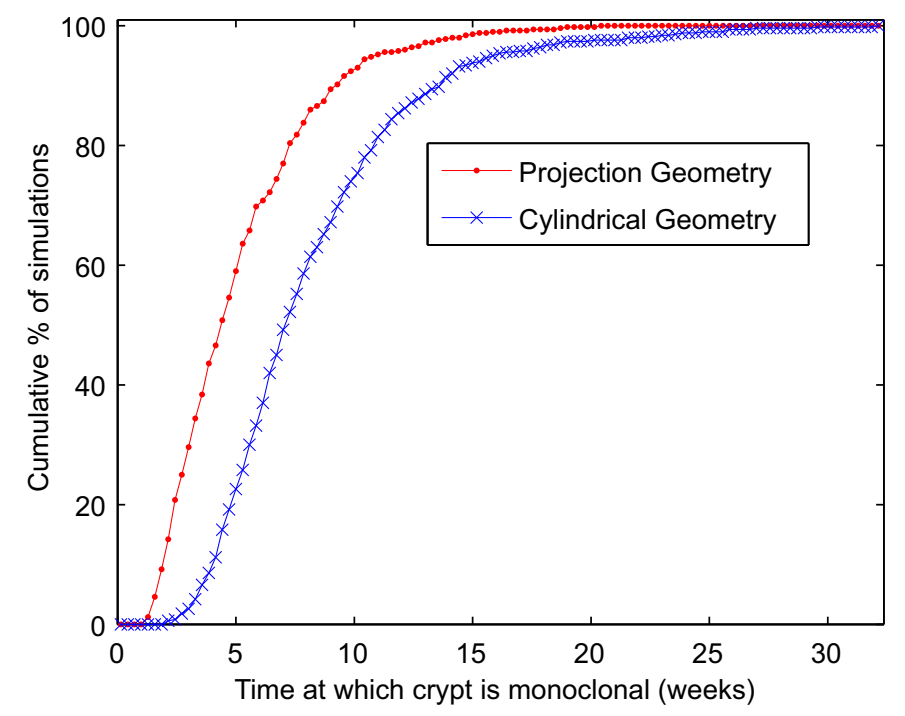

Fig. 17. Comparison of the cumulative distribution of monoclonal conversion times for the cylindrical and projection geometries for the case where cells in the niche have a longer mean G1 duration. The distributions were obtained by running 500 simulations for each geometry. Parameter values used in simulations are those given in Table 1 with $\mu_{\text {stem }}=14 \mathrm{~h}$ and $\mu_{\text {transit }}=2 \mathrm{~h}$.

linear equations

$\tau_{0}=0$,

$\tau_{i}=1+\sum_{j=1}^{\infty} p_{i j} \tau_{j}, \quad 1 \leq i \leq N$.

Solving the above equations, we find that

$\tau_{i}=\frac{i}{\alpha}\left(\frac{\alpha}{\frac{\alpha}{2}+\gamma(1-\alpha)}+2 N-1-i\right)$
In particular, the mean number of divisions before extinction occurs, starting with a single stem cell, is given by

$\tau_{1}=\frac{1}{\frac{\alpha}{2}+\gamma(1-\alpha)}+\frac{2}{\alpha}(N-1)$

Note that, as expected, $\tau_{1} \rightarrow \infty$ as $N \rightarrow \infty$, and that for fixed $N, \tau_{1}$ is a monotonic decreasing function of $\alpha$ in the interval $0<\alpha<1$.

\subsection{Alternative stochastic model of immortal stem cell hypothesis}

In the previous section we defined a time step to be the length of time between successive stem cell divisions. Although this makes it straightforward to compute properties such as the mean number of steps until extinction, mapping such a result back to actual units of time is problematic, since the average time between divisions is itself a function of the number of stem cells present. We therefore turn our attention to an alternative model, in which we consider 'generations' of stem cells, and initially make the simplifying assumption that these are contemporaneous. As before, our aim is to investigate the behavior of this process.

Let $Z_{n}$ denote the number of stem cells of a given clone in the $n$th generation, then

$Z_{n}=Y_{1}^{(n)}+\cdots+Y_{Z_{n-1}}^{(n)}$,

where $Y_{1}^{(n)}, \ldots, Y_{Z_{n-1}}^{(n)}$ are independent identically distributed random variables with common probability generating function

$G(s)=\frac{\alpha}{2}+(1-\alpha) s+\frac{\alpha}{2} s^{2}$.

We use the initial condition $Z_{0}=1$. We have for now neglected any upper bound on $Z_{n}$ to allow some progress with analysis of this process.

Let $G_{n}(s)=\mathbb{E}\left(s^{Z_{n}}\right)$ denote the generating function of $Z_{n}$. We can find a recurrence relation for the extinction probability $\rho_{n}=\mathbb{P}\left(Z_{n}=0\right)$ using the fact that $\rho_{n}=G_{n}(0)$ and the recurrence relation $G_{n}(s)=G\left(G_{n-1}(s)\right)$. Thus

$\rho_{n+1}=\frac{\alpha}{2}+(1-\alpha) \rho_{n}+\frac{\alpha}{2} \rho_{n}^{2}$,

for $n>0$, with $\rho_{0}=0$ and $\rho_{1}=\alpha / 2$. By employing the transformation $\rho_{n}=1-(2 / \alpha) v_{n}$, we find that this recurrence relation for $\rho_{n}$ is conjugate to the logistic map

$v_{n+1}=v_{n}\left(1-v_{n}\right)$,

for $n>0$, with $v_{1}=(\alpha / 2)(1-(\alpha / 2)) \in(0,1)$. It is known that the behavior of this map for a starting point in $(0,1)$ is monotonic convergence to zero as $n \rightarrow \infty$ (Drazin, 1992), and hence $\rho_{n}>1$ as $n \rightarrow \infty$. No closed-form solution for $\rho_{n}$ exists, however.

Now suppose that we start with $K$ clones, with populations $\left(Z_{n}^{(1)}\right)_{n \geq 0}, \ldots,\left(Z_{n}^{(K)}\right)_{n \geq 0}$, with each clone behaving as before. We wish to compute the expected time it takes for $K-1$ of these clones to become extinct.

The probability that by the $n$th generation we are left with $k$ clones is equal to the probability that the time to extinction is at most $n$ for $K-k$ of the clones, and is greater than $n$ for the remaining $k$ clones

$\mathbb{P}(k$ clones by $n$th generation $)=\left(\begin{array}{l}K \\ k\end{array}\right) \mathbb{P}(T \leq n)^{K-k}(1-\mathbb{P}(T \leq n))^{k}$

$$
=\left(\begin{array}{l}
K \\
k
\end{array}\right) \rho_{n}^{K-k}\left(1-\rho_{n}\right)^{k} .
$$

In particular, the probability of monoclonality by the $n$th generation is given by

$K \rho_{n}^{K-1}\left(1-\rho_{n}\right)$. 
While this result is valid for a population with contemporaneous generations, in most populations individuals in the same generation give birth to families at different times. This is certainly true in our stem cell example. To model this more complex behavior, we may attach another random variable to each individual, denoting their lifetime. Suppose that the collection of all stem cell lifetimes is a set of variables that are independent of each other and all family sizes, and are continuous and positive with common probability density function $f_{T}$. Each individual lives for a period of time equal to its lifetime before giving birth to its family of next-generation descendants.

Let $Z(t)$ denote the size of the population at time $t$. The generating function for the population size is $G_{t}(s)=\mathbb{E}\left(s^{Z(t)}\right)$. We assume that $Z(0)=1$ and let $T$ be the lifetime of this initial individual. Using conditional expectation, we have

$G_{t}(s)=\mathbb{E}\left(s^{Z(t)}\right)=\int_{0}^{\infty} \mathbb{E}\left(s^{Z(t)} \mid T=u\right) f_{T}(u) \mathrm{d} u$

If $u=T$, then at time $u$ the initial individual dies and is replaced by a random number $N$ of offspring, where $N$ has generating function $G$. Each of these offspring behaves in the future as their ancestor did in the past, and the effect of their ancestor's death is to replace the process by the sum of $N$ independent copies of the process displaced in time by an amount $u$.

If $u>t$, then $Z(t)=1$ and $\mathbb{E}\left(s^{Z(t)} \mid T=u\right)=s$. If $u<t$, then $Z(t)$ is the sum of $N$ independent copies of $Z(t-u)$, and so $\mathbb{E}\left(s^{Z(t)} \mid T=u\right)=$ $G\left(G_{t-u}(s)\right)$. We therefore have

$G_{t}(s)=\int_{0}^{t} G\left(G_{t-u}(s)\right) f_{T}(u) \mathrm{d} u+\int_{t}^{\infty} s f_{T}(u) \mathrm{d} u$

Unfortunately, this equation cannot be solved explicitly except in special cases. One such case is when $Z(t)$ is a Markov process, for example, when stem cell life times are exponentially distributed. In this case, if $T \sim \exp (\lambda)$, then it can be shown (Grimmett and Stirzaker, 1992) that Eq. (19) reduces to the partial differential equation

$\frac{\partial}{\partial t} G_{t}(s)=\lambda\left[G\left(G_{t}(s)\right)-G_{t}(s)\right]$.

However, this simplification may not be made for more realistic life-time distributions, such as those of stem cell populations in the crypt. In such cases Eq. (19) must be solved numerically.

As the above stochastic models demonstrate, a natural consequence of the immortal stem cell hypothesis is that each clonal population eventually hits zero with probability one. Thus every crypt will, eventually, lose all its stem cells. Using a simple stochastic model, we derived the estimate (12) for how the time scale over which this extinction occur depends on the size of the stem cell niche and the probability of symmetric division.

\subsection{Comparison of niche hypothesis results with stochastic model}

We now compare the results of our multiscale model to those of a much simpler non-spatial model, in which cell mechanics are neglected. Following Dingli et al. (2007), we make the simplifying assumption that the number of proliferating cells in the crypt, as well as the total number of cells in the crypt, do not change over time. This is true on average in the multiscale model, as demonstrated in Fig. 7. Under this assumption, we model the crypt dynamics by a Moran process. We separate the crypt into two compartments of constant size: a proliferative compartment of size $N_{p}$, consisting of all cells that are progressing through the cell cycle; and a non-proliferative compartment of size $N_{d}$, consisting of all cells that are differentiated. This simplified model assumes that all cells in each compartment are in equivalent positions, i.e. the compartment is well-mixed, and so allows us to study how important spatial effects are in determining the distribution of monoclonal conversion times.

Let $\left(X_{n}\right)_{n>0} \in\left\{0, \ldots, N_{p}\right\}$ denote the number of labeled cells in the proliferative compartment of the crypt after $n$ divisions, and let $\left(Y_{n}\right)_{n \geq 0} \in\left\{0, \ldots, N_{d}\right\}$ denote the number of labeled cells in the non-proliferative compartment of the crypt after $n$ divisions. We initially label a single proliferating cell with a harmless mutation, so that $X_{0}=1$ and $Y_{0}=0$, and track the progress of its progeny. At each time step, a cell is chosen at random from the proliferative compartment and divides, thus adding a new cell of the same type to the compartment. A cell is then chosen at random and moved from the proliferative compartment to the non-proliferative compartment. Lastly, a cell is chosen at random from the non-proliferative compartment and removed to simulate sloughing off the top of the crypt. This process ensures that both $N_{p}$ and $N_{d}$ remain constant over time. We assume that each time step is equal to $\tau / N_{p}$, where $\tau$ is the average cell cycle time for proliferating cells.

The transition probabilities $p_{i, j}=\mathbb{P}\left(X_{n+1}=j \mid X_{n}=i\right)$ are given by

$p_{i, i+1}=\frac{i\left(N_{p}-i\right)}{N_{p}\left(N_{p}+1\right)}$

$p_{i, i-1}=\frac{i\left(N_{p}-i\right)}{N_{p}\left(N_{p}+1\right)}$,

$p_{i, i}=\frac{i(i+1)+\left(N_{p}-i\right)\left(N_{p}+1-i\right)}{N_{p}\left(N_{p}+1\right)}$,

for $1 \leq i \leq N_{p}-1$, with $p_{0,0}=1$ and $p_{N, N}=1$. The transition probabilities $q_{i, j}=\mathbb{P}\left(Y_{n+1}=j \mid Y_{n}=i\right)$ are dependent on the current value of $X_{n}$, and are given by

$q_{i, i+1}=\frac{X_{n}\left(N_{d}-i\right)}{N_{p}\left(N_{d}+1\right)}$,

$q_{i, i-1}=\frac{\left(N_{p}-X_{n}\right) i}{N_{p}\left(N_{d}+1\right)}$

$q_{i, i}=\frac{X_{n}(i+1)+\left(N_{p}-X_{n}\right)\left(N_{d}-i\right)}{N_{p}\left(N_{d}+1\right)}$,

for $0 \leq i \leq N_{d}$.

Since all cells in the proliferative compartment are in equivalent positions, it is clear that the probability that the progeny of $i$ initial labeled cells ultimately take over the proliferative compartment, $\Phi_{i}$, is given by $i / N_{p}$. In particular, $\Phi_{1}=1 / N_{p}$, so we expect our single labeled cell to take over the proliferative compartment on average once every $N_{p}$ simulations. To verify this numerically, $10^{6}$ simulations of the model were performed with compartment sizes $N_{p}=150$ and $N_{d}=100$. The initial labeled cell was found to take over the proliferative compartment in 6672 simulations, compared with the theoretical value of 6666 .

The average time $T_{i}$ taken for the progeny of $i$ initial labeled cells to take over the proliferative compartment, conditional on this event occurring, satisfies the recurrence relation

$T_{i}=1+p_{i, i+1} T_{i+1}+p_{i, i} T_{i}+p_{i, i-1} T_{i-1}$,

for $1 \leq i \leq N_{p}-1$, with boundary conditions $T_{0}=0$ and $T_{N_{p}}=0$. Iterating, we find that

$T_{1}=\frac{\left(1-\Phi_{1}\right) R_{0}-R_{1}}{\Phi_{1}}$,

where

$R_{j}=\left(N_{p}+1\right) \sum_{i=j+1}^{N_{p}-1} \sum_{m=1}^{N_{p}-i} \frac{1}{m}$ 


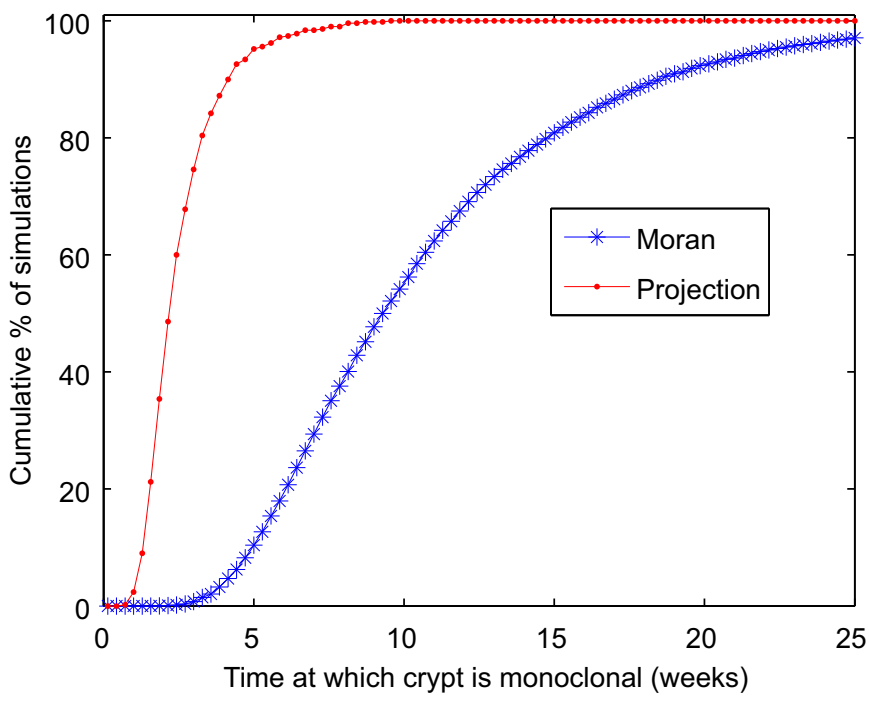

Fig. 18. Comparison of the cumulative distribution of monoclonal conversion times for the stochastic model and spatial model. The distributions were obtained by running 500 simulations for the spatial model and $10^{4}$ simulations for the stochastic model. Parameter values used in spatial simulations are those given in Table 1 with $\mu_{\text {stem }}=\mu_{\text {transit }}=2 \mathrm{~h}$.

We simulate the stochastic model, running $10^{4}$ simulations, and obtain a distribution of monoclonal conversion times that has mean 76.4 days and variance 1724.2 days. The theoretical expression for the mean time for a clonal population to take over the niche given by (20) gives $T_{1}=75$ days, which is extremely close to the distribution mean. Since $T_{1}$ does not include the time taken for a clonal population, once fixed in the niche, to take over the crypt, this suggests that this delay is not significant. Indeed, a rough estimate of the time taken for this to occur, assuming that the clonal population must take over the entire non-proliferative compartment, is given by $N_{d} \tau / N_{p} \sim 8$ h, i.e. less than a day.

The results of this stochastic model are compared to our spatial model in Fig. 18. To be able to compare like for like, we take $N_{p}=150$ and $\tau=12 \mathrm{~h}$ as in the spatial model. It is clear from Fig. 18 that the stochastic model leads to a gross overestimate of the time taken for a crypt to become monoclonal. This indicates that the key simplifying assumption made in the stochastic model, that each compartment is well-mixed, is not valid and that the spatial structure of the crypt is important. In particular, the fact that labeled cells are only lost from the edge of the proliferative compartment is neglected in the stochastic model, which results in an overestimate of the rate of loss of labeled cells and hence an underestimate in the net rate of growth of the labeled population.

\subsection{Comparison of results with experimental data}

We conclude with a comparison of our results to experimental data. As mentioned previously, Cook et al. (2000) used metallothionein immunopositivity following administration of the mutagen ENU to detect the frequency of murine colonic crypts wholly composed of mutated cells. This frequency can be increased in a dose-dependent manner after mutagen exposure. To a first approximation, we assume that the probability of accumulating a mutation is constant across crypts, and thus convert the frequency data obtained by Cook et al. (2000) to a cumulative distribution of the times taken for crypts to become monoclonal (consisting entirely of mutated cells) by dividing each data point by the maximum observed.

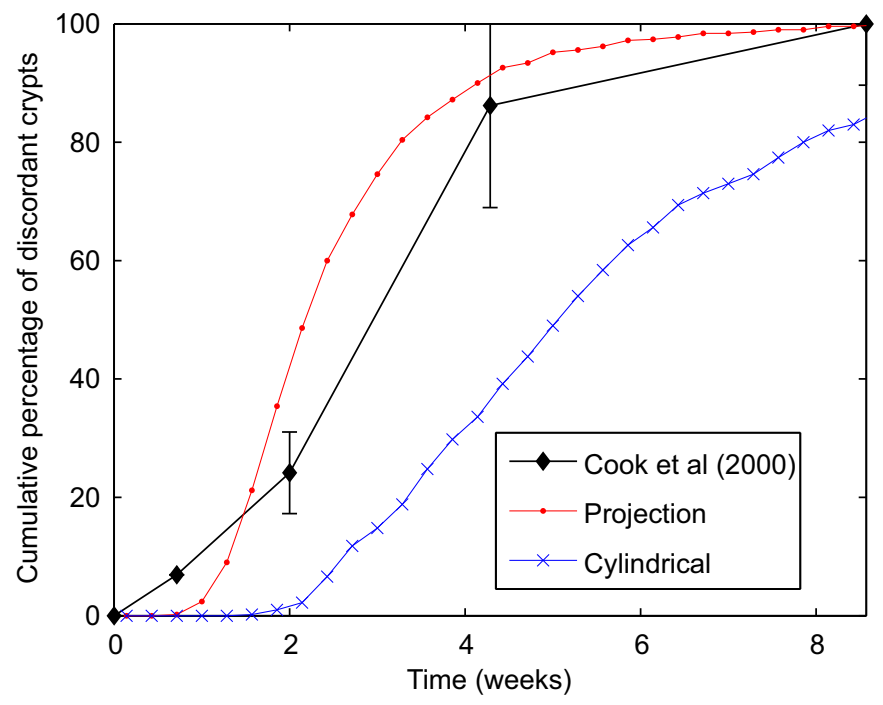

Fig. 19. Comparison of the results obtained by Cook et al. (2000) on the cumulative distribution of mutated crypts following adminstration of a mutagen with the theoretical distributions as predicted by the crypt projection model and the cylindrical crypt model. Error bars denote standard errors. Parameter values used in simulations are those given in Table 1 with $\mu_{\text {stem }}=\mu_{\text {transit }}=2 \mathrm{~h}$.

We compare these results to the cumulative distribution of monoclonal conversion times obtained using our crypt projection model under the stem cell niche hypothesis, and to those obtained using the cylindrical geometry employed by Meineke et al. (2001), in Fig. 19. We observe that the crypt projection model gives a much better fit to the data.

\section{Conclusions}

In this paper we have presented a novel spatial multiscale model of the colonic crypt. We have used our model to evaluate two different hypotheses concerning the behavior of crypt stem cells by studying the process of monoclonal conversion under each hypothesis. We found that the stem cell niche hypothesis, in which the proliferative capacity of each cell in the crypt is dictated by the local concentration of the Wnt growth factor, leads to more realistic results than the immortal stem cell hypothesis, in which certain cells at the base of the crypt are inherently stem cells, and each cell may divide a certain number of times before becoming terminally differentiated. In particular, we showed that the immortal stem cell hypothesis cannot lead to stable numbers of stem and transit cells without some form of population-dependent feedback. In contrast, under the stem cell niche hypothesis, a stable crypt architecture arises naturally. We also used our model to demonstrate that the spatial structure of the crypt has a large effect on the time scale over which a crypt becomes monoclonal, by comparing the model to a non-spatial stochastic model. The crypt geometry used in our model was also shown to be important, as our projection geometry gave much closer agreement to experimental data on monoclonal conversion times than the cylindrical geometry employed by Meineke et al. (2001).

When assuming the immortal stem cell hypothesis, we have not included any form of feedback which may stabilize the stem cell population in the crypt. It has been suggested that some form of contact inhibition operates, in which cells at the base of the crypt do not proliferate at their maximal rate due to overcrowding (van Leeuwen et al., 2009), giving rise to a complex pattern of cell proliferation up the crypt. This may be an avenue for future 
modeling. When assuming the stem cell niche hypothesis, we have superimposed a Wnt gradient. It is not clear precisely what form of gradient is needed to give rise to realistic crypt dynamics; Wnt may be secreted by cells residing at the base of the crypt, and diffuse up the crypt, or some other mechanism may operate. It would be interesting to study what effect different hypothetical mechanisms have on the model, in order to develop an integrative model that incorporates both asymmetric division and feedback mechanisms.

There are a number of avenues for future work. Several outstanding questions remain concerning the population dynamics of crypts that have accumulated cancerous mutations. It is known, for example, that mutations in key components of the Wnt pathway, such as APC and $\beta$-catenin, are the first step in colorectal carcinogenesis in the majority of colorectal cancers, including most hereditary cases (Ilyas, 2005). These mutations may affect the cell cycle, the distribution of growth factors, and cell-cell adhesion, all of which disrupt the otherwise tightly regulated crypt structure. Dingli et al. (2007) model the effect of APC mutations in a stochastic crypt model by introducing a relative reproductive fitness $r$ of mutant cells. Cells with a higher fitness either replicate faster, or generate more progeny in the same interval compared with other cells. At each time step cells are chosen randomly for division or removal, proportional to their fitness. In this model, APC mutations increase a cell's fitness, and also increase the probability that it undergoes self-renewal. A natural extension to our work is to investigate the effect of this type of mutation at various locations in the crypt. Another next step would be to remove the constraint on the geometry of the crypt. A model that incorporates a fully three-dimensional, deformable crypt geometry would allow us to investigate events that occur later in the colorectal carcinogenesis sequence, such as crypt buckling and crypt fission. This remains an avenue for future research.

\section{Acknowledgments}

The authors gratefully acknowledge funding through the Integrative Biology programme (GR/572023/01) and OCISB project (BB/D020190/1). AGF is supported by the EPRSC (EP/ I017909/1) and Microsoft Research, Cambridge.

\section{References}

Alberts, B., Johnson, A., Lewis, J., Raff, M., Roberts, K., Walter, P., 2002. Molecular Biology of the Cell, fourth ed. Garland Science.

Barker, N., van Es, J., Kuipers, J., Kujala, P., van den Born, M., Cozijnsen, M., Haegebarth, A., Korving, J., Begthel, H., Peters, P., Clevers, H., 2007. Identification of stem cells in small intestine and colon by marker gene Lgr5. Nature 449, 1003-1007.

Boman, B.M., Fields, J.Z., Bonham-Carter, O., Runquist, O.A., 2001. Computer modeling implicates stem cell overproduction in colon cancer initiation. Cancer Res. 61, 8408-8411.

Brittan, M., Wright, N., 2002. Gastrointestinal stem cells. J. Pathol. 197, 492-509.

Brittan, M., Wright, N., 2004. Stem cell in gastrointestinal structure and neoplastic development. Gut 53, 899-910.

Cancer Research UK, 〈http://info.cancerresearchuk.org/〉, 2010.

Cook, H., Williams, D., Thomas, G., 2000. Crypt-restricted metallothionein immunopositivity in murine colon: validation of a model for studies of somatic stem cell mutation. J. Pathol. 191, 306-312.

Dingli, D., Traulsen, A., Michor, F., 2007. (A)symmetric stem cell replication and cancer. PLOS Comput. Biol. 3, 482-487.

Drasdo, D., Loeffler, M., 2001. Individual-based models on growth and folding in one-layered tissues: intestinal crypts and early development. Nonlinear Anal. 47, 245-256.

Drazin, P., 1992. Nonlinear Systems. Cambridge University Press.

Gaspar, C., Fodde, R., 2004. APC dosage effects in tumorigenesis and stem cell differentiation. Int. J. Dev. Biol. 48, 377-386.
Giles, R., van Es, J., Clevers, H., 2003. Caught up in a Wnt storm: Wnt signaling in cancer. Biochim. Biophys. Acta 1653, 1-24.

Greaves, L., Preston, S., Tadrous, P., Taylor, R., Barron, M., Oukrif, D., Leedham, S. Deheragoda, M., Sasieni, P., Novelli, M., Jankowski, J., Turnbull, D., Wright, N., McDonald, S., 2006. Mitochondrial DNA mutations are established in human colonic stem cells, and mutated clones expand by crypt fission. Proc. Natl. Acad. Sci. USA 103, 714-719.

Grimmett, G., Stirzaker, D., 1992. Probability and Random Processes, second ed Oxford University Press.

Halm, D., Halm, S., 2000. Secretagogue response of goblet cells and columnar cells in human colonic crypts. Am. J. Physiol. Cell Physiol. 278, 212-233.

Ilyas, M., 2005. Wnt signalling and the mechanistic basis of tumour development J. Pathol. 205, 130-144

Johnston, M., Edwards, C., Bodmer, W., Maini, P., Chapman, S., 2007. Mathematica modeling of cell population dynamics in the colonic crypt and in colorectal cancer. Proc. Natl. Acad. Sci. USA 104, 4008-4013.

Kim, K., Shibata, D., 2002. Methylation reveals a niche: stem cell succession in human colon crypts. Oncogene 21, 5441-5449.

Korinek, V., Barker, N., Moerer, P., van Donselaar, E., Huls, G., Peters, P., Clevers, H. 1998. Depletion of epithelial stem-cell compartments in the small intestine of mice lacking Tcf-4. Nat. Genet. 19, 379-383.

Lechler, T., Fuchs, E., 2005. Asymmetric cell divisions promote stratification and differentiation of mammalian skin. Nature 437, 275-280.

Li, Y.Q., Roberts, S.A., Paulus, U., Loeffler, M., Potten, C.S., 1994. The crypt cycle in mouse small intestinal epithelium. J. Cell Sci. 107, 3271-3279.

Loeffler, M., Grossmann, B., 1991. A stochastic branching model with formation of subunits applied to the growth of intestinal crypts. J. Theor. Biol. 150, 175-191.

Loeffler, M., Stein, R., Wichmann, H.E., Potten, C.S., Kaur, P., Chwalinski, S., 1986 Intestinal crypt proliferation. I. A comprehensive model of steady-state proliferation in the crypt. Cell Tissue Kinet. 19, 627-645.

Loeffler, M., Birke, A., Pottern, C., 1993. Somatic mutation, monoclonality and stochastic models of stem cell organization in the intestinal crypt. J. Theor Biol. 160, 471-491.

Loeffler, M., Bratke, T., Paulus, U., Li, Y.Q., Potten, C.S., 1997. Clonality and life cycles of intestinal crypts explained by a state dependent stochastic model of epithelial stem cell organization. J. Theor. Biol. 186, 41-54.

Marshman, E., Booth, C., Potten, C., 2002. The intestinal epithelial stem cell. BioEssays 24, 91-98.

McDonald, S., Preston, S., Greaves, L., Leedham, S., Lovell, M., Jankowski, J., Turnbull, D., Wright, N., 2006. Clonal expansion in the human gut: mitochondrial DNA mutations show us the way. Cell Cycle 5, 808-811.

Meineke, F., Potten, C., Loeffler, M., 2001. Cell migration and organization in the intestinal crypt using a lattice-free model. Cell Prolif. 34, 253-266.

Norris, J., 1997. Markov Chains. Cambridge University Press.

Nowak, M., Komarova, N., Sengupta, A., Jallepalli, P., Shih, I.-M., Vogelstein, B. Langauer, C., 2002. The role of chromosomal instability in tumor initiation. Proc. Natl. Acad. Sci. USA 99, 16226-16231.

Okamoto, R., Watanabe, M., 2004. Molecular and clinical basis for the regeneration of human gastrointestinal epithelia. J. Gastroenterol. 39, 1-6.

Park, H., Goodlad, R., Wright, N., 1995. Crypt fission in the small intestine and colon. A mechanism for the emergence of G6PD locus-mutated crypts after treatment with mutagens. Am. J. Pathol. 147, 1416-1427.

Paulus, U., Potten, C., Loeffler, M., 1992. A model of the control of cellular regeneration in the intestinal crypt after perturbation based solely on local stem cell regulation. Cell Prolif. 25, 559-578.

Pitt-Francis, J., Pathmanathan, P., Bernabeu, M., Bordas, R., Cooper, J., Fletcher, A. Mirams, G., Murray, P., Osborne, J., Walter, A., Chapman, S., Garny, A. van Leeuwen, I., Maini, P., Rodriguez, B., Waters, S., Whiteley, J., Byrne, H. Gavaghan, D., 2009. Chaste: a test-driven approach to software development for biological modelling. Comput. Phys. Commun. 180, 2452-2471.

Potten, C.S., Loeffler, M., 1990. Stem cells: attributes, cycles, spirals, pitfalls and uncertainties. Lessons from the crypt. Development 110, 1001-1020.

Potten, C.S., Booth, C., Hargreaves, D., 2003. The small intestine as a model for evaluating adult tissue stem cell drug targets. Cell Prolif. 36, 115-129.

Preston, S., Wong, W., Chan, A., Poulsom, R., Jeffrey, R., Goodlad, R., Mandir, N., Elia, G., Novelli, M., Bodmer, W., Tomlinson, I., Wright, N., 2003. Bottom-up histogenesis of colorectal adenomas: origin in the monocryptal adenoma and initial expansion by crypt fission. Cancer Res. 63, 3819-3825.

Reya, T., Morrison, S., Clarke, M., Weissman, I., 2001. Stem cells, cancer, and cancer stem cells. Nature 414, 105-111.

Ro, S., Rannala, B., 2001. Methylation patterns and mathematical models reveal dynamics of stem cell turnover in the human colon. Proc. Natl. Acad. Sci. USA 98, 10519-10521.

Roeder, I., Lorenz, R., 2006. Asymmetry of stem cell fate and the potential impact of the niche: observations, simulations, and interpretations. Stem Cell Rev. 2, 171-180.

Ross, M., Kaye, G., Pawlina, W., 2003. Histology: A Text and Atlas. Lippincott Williams \& Wilkins.

Sansom, O., Reed, K., Hayes, A., Ireland, H., Brinkmann, H., Newton, I., Batlle, E., Simon-Assman, P., Clevers, H., Nathke, I., Clarke, A., Winton, D., 2004. Loss of Apc in vivo immediately perturbs Wnt signaling, differentiation, and migration. Genes Dev. 18, 1385-1390.

Schofield, R., 1978. The relationship between the spleen colony-forming cell and the haemopoietic stem cell. Blood Cells 4, 7-25.

Spradling, A., Drummond-Barboa, D., Kai, T., 2001. Stem cells find their niche. Nature 414, 98-104. 
Tyson, J., Chen, K., Novák, B., 2001. Network dynamics and cell physiology. Nat Rev. Mol. Cell Biol. 2, 908-916.

Tyson, J., Csikász-Nagy, A., Novák, B., 2002. The dynamics of cell cycle regulation. BioEssays 24, 1095-1109.

van de Wetering, M., Sancho, E., Verweij, C., de Lau, W., Oving, I., Hurlstone, A., van der Horn, K., Batlle, E., Coudreuse, D., Haramis, A., Tjon-Pon-Fong, M., Moerer, P., van den Born, M., Soete, G., Pals, S., Eilers, M., Medema, R., Clevers, H., 2002. The $\beta$-catenin/TCF-4 complex imposes a crypt progenitor phenotype on colorectal cancer cells. Cell 111, 241-250.

van Leeuwen, I., Mirams, G., Walter, A., Fletcher, A., Murray, P., Osborne, J., Varma, S. Young, S., Cooper, J., Doyle, B., Pitt-Francis, J., Momtahan, L., Pathmanathan, P., Whiteley, J., Chapman, S., Gavaghan, D., Jensen, O., King, J., Maini, P., Waters, S.
Byrne, H., 2009. An integrative computational model for intestinal tissue renewal. Cell Prolif. 42, 617-636.

Williams, E., Lowes, A., Williams, D., Williams, G., 1992. A stem cell niche theory of intestinal crypt maintenance based on a study of somatic mutation in colonic mucosa. Am. J. Pathol. 141, 773-776.

Winton, D., Ponder, B., 1990. Stem-cell organization in mouse small intestine. Proc. R. Soc. Lond. B 241, 13-18.

Winton, D., Blount, M., Ponder, B., 1988. A clonal marker induced by mutation in mouse intestinal epithelium. Nature 333, 463-466.

World Health Organization, 〈http://www.who.int/topics/ageing/en 〉, 2008.

Yatabe, Y., Tavaré, S., Shibata, D., 2001. Investigating stem cells in human colon by using methylation patterns. Proc. Natl. Acad. Sci. USA 98, 10839-10844. 